\title{
Differential Modular Forms on Shimura Curves, I
}

\author{
ALEXANDRU BUIUM \\ University of New Mexico, Albuquerque, NM 87131, U.S.A.e-mail: buium@math.unm.edu
}

(Received: 11 February 2002; accepted in final form: 14 August 2002)

\begin{abstract}
The quotient of a Shimura curve by the isogeny equivalence relation is not an object of algebraic geometry. The paper shows how this quotient space becomes a geometric object in a more general geometry obtained from 'usual algebraic geometry', by adjoining a new operation; this operation looks like a 'Fermat quotient' and should be viewed as an arithmetic analogue of usual derivations.
\end{abstract}

Mathematics Subject Classifications (2000). Primary: 14-XX; Secondary: 11-XX.

Key words. isogeny, modular forms, Shimura curves.

\section{Main Concepts and Results}

\subsection{INTRODUCTION}

The quotient of an algebraic variety $X$ by a Zariski dense equivalence relation $E \subset X \times X$ is not, in any reasonable sense, an object of usual algebraic geometry. Typical examples of Zariski dense equivalence relations that one would like to study are: (1) the isogeny equivalence relation on various moduli spaces of Abelian varieties equipped with various polarization, level, and endomorphism structures and (2) the orbit equivalence relation on a variety equipped with an endomorphism, the latter being the paradigm for algebraic discrete dynamical systems. In a number of previous papers the author used two extensions of algebraic geometry, which can be called differential algebraic geometries, in order to study quotients by Zariski dense equivalence. The two extensions are: (i) the Ritt-Kolchin geometry [4, 21, 28] obtained by adjoining, to usual algebraic geometry, a derivation operator, and (ii) an arithmetic analogue of the Ritt-Kolchin geometry that was initiated in [5, 6], which is obtained by adjoining, to usual algebraic geometry, the Fermat quotient operator. We shall be interested here in this arithmetic analogue.

Quotients of modular curves (or more generally of moduli spaces of Abelian varieties) by the isogeny equivalence relation were studied in $[1,2,7]$. One can map these quotients into projective spaces using what we call isogeny covariant differential modular forms in a way similar to that in which modular curves are embedded into projective spaces by means of usual modular forms. In this paper we develop a similar theory for Shimura curves associated to indefinite, non-split quaternion algebras $D$ over $\mathbf{Q}$. Although the results for Shimura curves will be similar to the ones for 
modular curves, the methods of proof will be sometimes quite different; cf. the remarks below. One of our main results (Theorem 1.2) will state that all the spaces of isogeny covariant differential modular forms of a given weight, for a given Shimura curve, are finite dimensional; for certain basic weights we will find that this space has rank one, a situation similar to what happens for modular curves; cf. [7] and [1]. Another, rather surprising, result (Theorem 1.6) will say that a certain natural ring of isogeny covariant differential modular forms for a Shimura curve is isomorphic to the corresponding ring for the modular curves. This can be viewed as an instance of the analogy between modular curves and Shimura curves.

As explained above quotients of modular curves and Shimura curves by the isogeny equivalence relation are just examples of a more general theory. Another example of that theory, namely spaces of orbits of discrete dynamical systems, will be investigated in [11]. A general formalism that embraces all these examples is explained in [10]. One should also note that, in different contexts (that include differentiable dynamical systems), Alain Connes [13] considered a noncommutative enlargement of usual geometry in order to construct quotients which do not exist in point set geometry.

Our strategy in the case of modular curves and moduli spaces of Abelian varieties $[1,2,7]$, was based on Fourier expansions, i.e. expansions in the Fourier-Tate $q$-parameter at a cusp; cf. [20]. Since Shimura curves don't have cusps, Fourier expansions are not available for them. As a substitute we shall use Serre-Tate expansions; these are expansions in the Serre-Tate $q$-parameters at ordinary points [19] and were studied, for usual (i.e. nondifferential) modular forms, by A. Mori in a beautiful series of papers; cf. [25] and the papers cited therein. (We will not need here any of Mori's results, but some of our preparatory discussion on Serre-Tate parameters for Shimura curves overlaps with similar discussion in Mori's papers. Note, however, that the normalizations most convenient for our purposes here differ from Mori's so, for the convenience of the reader, we shall redo some of the basics on Serre-Tate parameters in our context.) Here is a comment which might be useful for the non-expert reader. The Serre-Tate $q$-parameter on a modular curve (proved to be equal to Dwork's $q$-parameter by Katz [19]) is a priori unrelated to the Fourier-Tate $q$-parameter; the former tells the story away from the cusps whereas the latter tells the story at the cusps. It comes, therefore, as a surprise to see, as we shall, that the Fourier and the Serre-Tate expansions (formally) coincide for a remarkable class of isogeny covariant differential modular forms on modular curves, or more generally, for a certain class of isogeny covariant Siegel differential modular forms; cf. Theorem 2.3.

Here is the plan of the paper. We start by quickly reviewing (and setting notations for) quaternion algebras $[3,12,30]$, false elliptic curves $[3,12,15]$, and Siegel differential modular forms [2]. Then we define differential modular forms on indefinite, non-split quaternion algebras $D$ over $\mathbf{Q}$ and state our main results; cf. the end of the present Section 1. In Section 2 we develop our main tool, the Serre-Tate expansions of (Siegel) differential modular forms, and we establish the link between SerreTate and Fourier expansions of certain such forms. We also prove that there is an 
isomorphism between a certain natural ring of differential modular forms on $D$ and the corresponding ring for the modular curve. In Section 3 we show that the Serre-Tate expansions of isogeny covariant differential modular forms on $D$, of a given weight, satisfy a certain functional equation. Then we show that the space of series that satisfy this equation is finite-dimensional. This plus a differential Serre-Tate $q$-expansion principle will conclude our proof that the space of isogeny covariant differential modular forms on $D$, of a given weight, is finitely generated and will also conclude the proof of one of our rank one results in Theorem 1.2. Another rank one result claimed in that Theorem will require considerable extra effort to prove; this will be done in a sequel to the present paper [9]. By analogy with [1] we will develop in [9] an analogue of Serre's differential operators in our context; however, unlike in [1] (where the explicit structure of the ring of classical modular forms of level one was used through computations in the 'coordinates' $E_{4}$ and $E_{6}$ ) we will need to adopt in [9] a coordinate free approach.

\subsection{QUATERNION ALGEBRAS $([3,12,30])$}

Let $D$ be a quaternion algebra over $\mathbf{Q}$; recall that this means, by definition, that $D$ is a ring whose center is $\mathbf{Q}$, such that $D$ has dimension 4 as a $\mathbf{Q}$-vector space, and $D$ has no nontrivial two-sided ideals. Assume $D$ is nonsplit (i.e. $D \nsucceq \operatorname{Mat}_{2}(\mathbf{Q})$; equivalently $D$ is a division ring) and $D$ is indefinite (i.e. $D \otimes_{\mathbf{Q}} \mathbf{R} \simeq \operatorname{Mat}_{2}(\mathbf{R})$ ). Let $d=\operatorname{disc}(D)$ be the discriminant of $D$ (i.e. $d$ is the product of all the primes $l$ for which $D \otimes_{\mathbf{Q}} \mathbf{Q}_{l} \nsucc \operatorname{Mat}_{2}\left(\mathbf{Q}_{l}\right)$.) Let $F$ be a field of characteristic zero such that we have an isomorphism

$$
j: D \otimes_{\mathbf{Q}} F \rightarrow \operatorname{Mat}_{2}(F) .
$$

(For instance, $F$ can be $\overline{\mathbf{Q}}, \mathbf{R}$, or $\mathbf{Q}_{p}$ for $p$ not dividing the discriminant $d$.) For any $\alpha \in D$ consider the reduced trace and norm: $\operatorname{tr}(\alpha):=\operatorname{tr}(j(\alpha)) \in F, \operatorname{det}(\alpha):=$ $\operatorname{det}(j(\alpha)) \in F$. It is known that $\operatorname{tr}(\alpha), \operatorname{det}(\alpha)$ actually belong to $\mathbf{Q}$ and are independent of the choice of $F$ and $K$. On the other hand, we have at our disposal the usual trace and norm of the $\mathbf{Q}$-algebra $D: \operatorname{tr}_{D / \mathbf{Q}}, N_{D / \mathbf{Q}}: D \rightarrow \mathbf{Q}$. The following formulae hold:

$$
\operatorname{tr}_{D / \mathbf{Q}}(\alpha)=2 \cdot \operatorname{tr}(\alpha), \quad N_{D / \mathbf{Q}}(\alpha)=\operatorname{det}(\alpha)^{2} \quad(\alpha \in D) .
$$

Consider now the canonical involution

$$
D \rightarrow D, \quad \alpha \mapsto \alpha^{\prime}:=\operatorname{det}(\alpha) \cdot \alpha^{-1} \quad(\alpha \neq 0)
$$

So if $j(\alpha)=\left[\begin{array}{ll}a & b \\ c & d\end{array}\right]$, then $j\left(\alpha^{\prime}\right)=\left[\begin{array}{cc}d & -b \\ -c & a\end{array}\right]$. There exists a maximal order $\mathcal{O}_{D}$ of $D$ (i.e. a maximal element in the set of all subrings $\mathcal{O} \subset D$ with $\mathcal{O} \simeq \mathbf{Z}^{4}$ as $\mathbf{Z}$-modules and $\mathcal{O} \otimes_{\mathbf{Z}} \mathbf{Q}=D$ ) which is stable under the canonical involution $\alpha \mapsto \alpha^{\prime}$. Since for $\alpha \in \mathcal{O}_{D}$ we have $\operatorname{tr}_{D / \mathbf{Q}}(\alpha), N_{D / \mathbf{Q}}(\alpha) \in \mathbf{Z}$ it follows that

$$
\operatorname{tr}(\alpha) \in \frac{1}{2} \mathbf{Z}, \quad \operatorname{det}(\alpha) \in \mathbf{Z} \quad\left(\alpha \in \mathcal{O}_{D}\right) .
$$


It is known that there exists an element $\tau \in \mathcal{O}_{D}$ such that $\tau^{2}=-d$. Note that $\left(\tau^{\prime}\right)^{2}=-d$, hence, since $\tau, \tau^{\prime}$ commute and $D$ is a division ring, $\tau^{\prime}=-\tau$. Also $\tau^{-1}=-(1 / d) \tau$. A key role in the theory is played by the involution

$$
D \rightarrow D, \quad \alpha \mapsto \alpha^{+}:=\tau^{-1} \alpha^{\prime} \tau=\tau \alpha^{\prime} \tau^{-1},
$$

which is known to induce a nontrivial involution of $\mathcal{O}_{D}$.

Let us fix a prime $p \geqslant 5$ that does not divide $d$ and let $F=\mathbf{Q}_{p}$. Since $\mathcal{O}_{D} \otimes_{\mathbf{Z}} \mathbf{Z}_{p}$ is a maximal order in $D \otimes_{\mathbf{Q}} \mathbf{Q}_{p}$ it follows that $j\left(\mathcal{O}_{D} \otimes_{\mathbf{Z}} \mathbf{Z}_{p}\right)$ is a maximal order in $\operatorname{Mat}_{2}\left(\mathbf{Q}_{p}\right)$. Since any maximal order of $\operatorname{Mat}_{2}\left(\mathbf{Q}_{p}\right)$ is conjugated to $\operatorname{Mat}_{2}\left(\mathbf{Z}_{p}\right)$ we may (and will) assume, by composing $j$ with an inner automorphism of $\operatorname{Mat}_{2}\left(\mathbf{Q}_{p}\right)$, that $j\left(\mathcal{O}_{D} \otimes_{\mathbf{Z}} \mathbf{Z}_{p}\right)=\operatorname{Mat}_{2}\left(\mathbf{Z}_{p}\right)$. So we have an isomorphism

$$
j: \mathcal{O}_{D} \otimes \mathbf{Z} \mathbf{Z}_{p} \rightarrow \operatorname{Mat}_{2}\left(\mathbf{Z}_{p}\right) .
$$

Now we have the following easy lemma:

LEMMA 1.1. If $A \in \operatorname{Mat}_{2}\left(\mathbf{Z}_{p}\right)$ is a nonscalar matrix such that $A^{2}=m I$ with $m \in \mathbf{Z}_{p}^{\times}$ (and I the identity), then there exists $U \in \mathrm{GL}_{2}\left(\mathbf{Z}_{p}\right)$ such that

$$
U A U^{-1}=\left[\begin{array}{cc}
0 & 1 \\
m & 0
\end{array}\right]
$$

Proof. Since $A^{2}$ is scalar while $A$ is not it is easy to check that $A$ cannot be $\equiv \bmod$ $p$ to a scalar matrix. Then one easily checks that for $v$ appropriately chosen in the set

$$
\left\{\left[\begin{array}{l}
1 \\
0
\end{array}\right],\left[\begin{array}{l}
0 \\
1
\end{array}\right],\left[\begin{array}{l}
1 \\
1
\end{array}\right]\right\}
$$

the set $\{v, A v\}$ is a basis of $\mathbf{Z}_{p}^{2}$ and we are done.

So we can (and will) assume that (1.2) is such that

$$
j(\tau)=\left[\begin{array}{cc}
0 & 1 \\
-d & 0
\end{array}\right]
$$

Throughout the paper we shall fix the following data:

$$
D, \mathcal{O}_{D}, p, F, j, \tau,
$$

where $F=\mathbf{Q}_{p}$.

\subsection{ISOGENIES [2]}

For any Noetherian ring $S$ we let $\mathbf{M}_{g}(S)$ denote the set of all triples $(A, \theta, \omega)$ where $A / S$ is an Abelian scheme of relative dimension $g, \theta: A \rightarrow \check{A}$ is a principal polarization, and $\omega=\left(\omega_{1}, \ldots, \omega_{g}\right)^{t}$ is a column vector whose entries are a basis of the $S$-module of 1-forms $H^{0}\left(A, \Omega_{A / S}^{1}\right)$; so the latter is supposed, by our very definition, to be free. Let

$$
\left(A_{1}, \theta_{1}, \omega_{1}\right),\left(A_{2}, \theta_{2}, \omega_{2}\right) \in \mathbf{M}_{g}(S)
$$


and let $u: A_{1} \rightarrow A_{2}$ be an isogeny (i.e. a finite flat homomorphism; our isogenies are not assumed to be compatible with the forms or the polarizations). We let

$$
u^{t}:=\theta_{1}^{-1} \circ \check{u} \circ \theta_{2}: A_{2} \rightarrow \check{A}_{2} \rightarrow \check{A}_{1} \rightarrow A_{1}
$$

denote its transpose and we let $[u] \in \operatorname{Mat}_{g}(S)$ be the unique matrix such that $u^{*} \omega_{2}=[u] \cdot \omega_{1}$. Note that $u^{t t}=u$ and $[u \circ v]=[u][v]$. (If $A_{1}=A_{2}=A, \theta_{1}=\theta_{2}=\theta$, then $u \mapsto u^{t}$ is the Rosati involution of $(A, \theta)$.) Let

$$
\mathrm{d}(u):=\operatorname{det}\left(\left[u \circ u^{t}\right]\right)=\operatorname{det}([u]) \operatorname{det}\left(\left[u^{t}\right]\right) \in S .
$$

If $g=1$ we have $d(u)=\operatorname{deg}(u)$, the degree of $u$. More generally, by [2], Lemma 1.1,

$$
\operatorname{deg}(u)^{2}=\mathrm{d}(u)^{2} .
$$

In particular, if $S$ is a $\mathbf{Z}_{p}$-algebra then $\operatorname{deg}(u)$ is prime to $p$ if and only if $u$ and $\check{u}$ are etale; in this case, $[u],\left[u^{t}\right] \in \mathrm{GL}_{g}(S)$; also, since $\operatorname{deg}(u)$ is locally constant, so is $\mathrm{d}(u)$.

\subsection{FALSE ELLIPTIC CURVES $([3,12,15])$}

Let $S$ be a Noetherian (commutative) $\mathbf{Z}_{p}$-algebra. By a false elliptic curve over $S$ we mean a pair $(A, i)$ where $A / S$ is an Abelian scheme of relative dimension two, and $i: \mathcal{O}_{D} \rightarrow \operatorname{End}(A / S)$ is an injective ring homomorphism (preserving the unit element). Since $D$ is a division algebra $i(\alpha)$ is automatically finite for all $\alpha \neq 0$. So any such $i(\alpha)$ is finite and flat fiber by fiber. So $i(\alpha)$ itself is flat, hence an isogeny, for all $\alpha \neq 0$. Cf. the argument in [22] p. 77.

For any principal polarization $\theta: A \rightarrow \check{A}$ we have defined a Rosati involution

$$
\operatorname{End}(A / S) \rightarrow \operatorname{End}(A / S), \quad u \mapsto u^{t}:=\theta^{-1} \circ \check{u} \circ \theta .
$$

By a polarized false elliptic curve over $S$ we will understand a triple $(A, i, \theta)$ where $(A, i)$ is a false elliptic curve and $\theta: A \rightarrow \breve{A}$ is a principal polarization such that the Rosati involution 1.5 on $\operatorname{End}(A / S)$ induces the involution $\alpha \mapsto \alpha^{+}$on $\mathcal{O}_{D}$. By [12] if $(A, i)$ is a false elliptic curve then there is a unique principal polarization $\theta$ on $A$ such that for any geometric point $\bar{s}$ of $S$ the Rosati involution on $\operatorname{End}\left(A_{\bar{s}}\right)$ is compatible with the involution $\alpha \mapsto \alpha^{+}$on $\mathcal{O}_{D}$. So if $\left(A, i, \theta_{1}\right)$ and $\left(A, i, \theta_{2}\right)$ are polarized false elliptic curves then $\theta_{1}=\theta_{2}$. Also, if $S$ is reduced then for any false elliptic curve $(A, i)$ there is a (unique) $\theta$ such that $(A, i, \theta)$ is a polarized false elliptic curve. Let $\left(A_{1}, i_{1}, \theta_{1}\right),\left(A_{2}, i_{2}, \theta_{2}\right)$ be polarized false elliptic curves; by an isogeny of false polarized elliptic curves we mean an isogeny $u: A_{1} \rightarrow A_{2}$ over $S$ such that $i_{2}(\alpha) \circ u=u \circ i_{1}(\alpha)$ for all $\alpha \in \mathcal{O}_{D}$. (Note that $i_{1}(\alpha)$ for $\alpha \in \mathcal{O}_{D}, \alpha \neq 0$, is, by definition, an isogeny of Abelian schemes but it is not an isogeny of polarized false elliptic curves, unless $\alpha$ is in the center of $D$ !) One knows that

$$
u^{t}:=\theta_{1}^{-1} \circ \check{u} \circ \theta_{2}: A_{2} \rightarrow A_{1}
$$

is also an isogeny of false polarized elliptic curves and the composition $\partial(u):=u^{t} \circ u$ is (the multiplication by) an integer called the false degree of $u$. The actual degree $\operatorname{deg}(u)$ of $u$ is trivially seen to be $\partial(u)^{2}$. 
By a false 1-form $\omega$ on a false polarized elliptic curve $(A, i, \theta)$ we will understand a column vector $\omega=\left(\omega^{1}, \omega^{2}\right)^{t}$ where $\omega^{1}, \omega^{2} \in H^{10}:=H^{0}\left(A, \Omega_{A / S}\right)$ such that

$$
i(\alpha)^{*} \omega=j(\alpha) \omega, \quad \alpha \in \mathcal{O}_{D} .
$$

(So, if one views $i(\alpha)$ as an isogeny from $(A, \theta, \omega)$ to itself, then $[i(\alpha)]=j(\alpha)$ for all $\alpha \in \mathcal{O}_{D}$.) We say an $\omega$ as above is invertible if $\omega^{1}, \omega^{2}$ form a basis of the $S$-module $H^{10}$. It is trivial to check that if $\omega$ is a false invertible 1 -form then $\omega$ is a basis for the $S$-module of false 1 -forms. Also, if $u: A_{1} \rightarrow A_{2}$ is an isogeny of polarized false elliptic curves $\left(A_{1}, i_{1}, \theta_{1}\right)$ and $\left(A_{2}, i_{2}, \theta_{2}\right)$ whose degree is not divisible by $p$ and if $\omega$ is a false invertible 1 -form on $\left(A_{2}, i_{2}, \theta_{2}\right)$ then $u^{*} \omega$ is a false invertible 1-form on $\left(A_{1}, i_{1}, \theta_{1}\right)$.

Note that if $(A, i, \theta)$ is any polarized false elliptic curve over $S$ then the $S$-module of false 1-forms has a useful alternative description as follows. We have a natural ring homomorphism

$$
\mathcal{O}_{D}^{o p} \rightarrow \operatorname{End}_{S}\left(H^{10}\right), \quad \alpha \mapsto i(\alpha)^{*}
$$

which induces a ring homomorphism

$$
l: \mathcal{O}_{D}^{o p} \otimes_{\mathbf{z}} S \rightarrow \operatorname{End}_{S}\left(H^{10}\right) .
$$

On the other hand, the isomorphism

$$
j: \mathcal{O}_{D} \otimes \mathbf{Z} \mathbf{Z}_{p} \rightarrow \operatorname{Mat}_{2}\left(\mathbf{Z}_{p}\right)
$$

induces an isomorphism

$$
j: \mathcal{O}_{D}^{o p} \otimes_{\mathbf{Z}} S \rightarrow \operatorname{Mat}_{2}(S)^{o p} .
$$

Consider the ring homomorphism

$$
l \circ j^{-1}: \operatorname{Mat}_{2}(S)^{o p} \rightarrow \mathcal{O}_{D}^{o p} \otimes_{\mathbf{Z}} S \rightarrow \operatorname{End}_{S}\left(H^{10}\right) .
$$

Let $e_{11}, e_{12}, e_{21}, e_{22}$ be the images, via 1.9 of the matrices

$$
\left[\begin{array}{ll}
1 & 0 \\
0 & 0
\end{array}\right],\left[\begin{array}{ll}
0 & 1 \\
0 & 0
\end{array}\right],\left[\begin{array}{ll}
0 & 0 \\
1 & 0
\end{array}\right],\left[\begin{array}{ll}
0 & 0 \\
0 & 1
\end{array}\right] .
$$

Then we have a direct sum decomposition

$$
H^{10}=e_{11} H^{10} \oplus e_{22} H^{10}
$$

hence $e_{11} H^{10}$ is a locally free $S$-module of rank one. We claim that $e_{11} H^{10}$ is isomorphic to the $S$-module of false 1 -forms. Indeed, if $\omega^{1} \in e_{11} H^{10}$ it is trivial to see that $\omega^{2}:=e_{12} \omega^{1} \in e_{22} H^{10}$ and the column vector $\omega:=\left(\omega^{1}, \omega^{2}\right)$ is a false 1 -form. Conversely, if $\left(\omega^{1}, \omega^{2}\right)^{t}$ is a false 1-form then $\omega^{1} \in e_{11} H^{10}$. The two maps, described above, between $e_{11} H^{10}$ and the module of false 1-forms are inverse to each other. (By the way, if $e_{11} H^{10}$ happens to be free, which is the case locally in the Zariski topology, and $\omega^{1}$ is a basis for this module, then it is trivial to check that $\omega^{2}:=e_{12} \omega^{1}$ is a basis for $e_{22} H^{10}$, hence $\left(\omega^{1}, \omega^{2}\right)$ is an invertible false 1 -form. Hence, 
if $\omega$ is a basis for the module of false 1 -forms then $\omega$ is invertible. We conclude that invertible false 1-forms always exist locally in the Zariski topology.)

Denote, in what follows, by $\mathbf{M}_{D}(S)$ the set of all tuples $(A, i, \theta, \omega)$ where $(A, i, \theta)$ is a polarized false elliptic curve over $S$ and $\omega$ is a false invertible 1-form on it. (Recall that $\mathbf{M}_{D}(S)$ implicitly depends on all the data (1.3) and not merely on $D$.)

Clearly, using the notations in the previous subsection, we have a 'forgetful map':

$$
\mathbf{M}_{D}(S) \rightarrow \mathbf{M}_{2}(S), \quad(A, i, \theta, \omega) \mapsto(A, \theta, \omega) .
$$

Let $\left(A_{1}, i_{1}, \theta_{1}, \omega_{1}\right),\left(A_{2}, i_{2}, \theta_{2}, \omega_{2}\right) \in \mathbf{M}_{D}(S)$ and let $u: A_{1} \rightarrow A_{2}$ be an isogeny of false elliptic curves. Since $u^{*} \omega_{2}$ is a false invertible 1 -form the matrix $[u] \in \operatorname{Mat}_{2}(S)$ must be a scalar matrix. Similarly, $\left[u^{t}\right]$ is a scalar matrix. Moreover, since $u^{t} \circ u=\partial(u)$ we have $\left[u^{t}\right][u]=\partial(u) \cdot I$. Hence, $d(u)=\operatorname{det}(\partial(u) \cdot I)=\partial(u)^{2}=$ $\operatorname{deg}(u)$.

We will also need the following degree computation. Let $(A, i, \theta, \omega) \in \mathbf{M}(S)$ and let $\alpha \in \mathcal{O}_{D}, \alpha \neq 0$. We claim that

$$
\operatorname{deg}(i(\alpha))=\operatorname{det}(\alpha)^{2} \text {. }
$$

Indeed we have

$$
\begin{aligned}
\mathrm{d}(i(\alpha)) & =\operatorname{det}([i(\alpha)]) \cdot \operatorname{det}\left(\left[i(\alpha)^{t}\right]=\operatorname{det}([i(\alpha)]) \cdot \operatorname{det}\left(\left[i\left(\alpha^{+}\right)\right]\right.\right. \\
& =\operatorname{det}(\alpha) \cdot \operatorname{det}\left(\alpha^{+}\right)=\operatorname{det}(\alpha) \cdot \operatorname{det}\left(\alpha^{\prime}\right)=\operatorname{det}(\alpha)^{2} .
\end{aligned}
$$

Hence $\operatorname{deg}(i(\alpha))^{2}=\mathrm{d}(i(\alpha))^{2}=\operatorname{det}(\alpha)^{4}$ and our claim is proved.

\subsection{PROLONGATIONS [2]}

The ^ sign will always denote $p$-adic completion. A module $M$ over a ring will be called $p$-adically complete if $\hat{M}=M$. Recall from [7] that a $p$ derivation $\delta: A \rightarrow B$ from a ring $A$ into an $A$-algebra $B$ is a map satisfying

$$
\begin{aligned}
& \delta(x+y)=\delta x+\delta y+C_{p}(x, y), \\
& \delta(x y)=x^{p} \delta y+y^{p} \delta x+p \delta x \delta y,
\end{aligned}
$$

where $C_{p}(X, Y):=\left(X^{p}+Y^{p}-(X+Y)^{p}\right) / p \in \mathbf{Z}[X, Y]$. If $\delta$ is a $p$-derivation then the map $\phi: A \rightarrow B$ defined by $\phi(x):=x^{p}+p \delta x$ is a ring homomorphism. By a prolongation sequence we mean a sequence of rings $S^{n}, n=0,1,2, \ldots$, such that each $S^{n+1}$ is an $S^{n}$-algebra and such that one is given $p$-derivations $\delta_{n}: S^{n} \rightarrow S^{n+1}$, each $\delta_{n}$ prolonging, in the obvious sense, $\delta_{n-1}$. By abuse we shall denote all $\delta_{n}$ 's by the same letter, $\delta$, and we shall denote prolongation sequences as above by $S^{*}$. A morphism of prolongation sequences $S^{*} \rightarrow \tilde{S}^{*}$ is simply a sequence of ring homomorphisms $\pi_{n}: S^{n} \rightarrow \tilde{S}^{n}$ which is compatible with the structure algebra homomorphisms in both $S^{*}$ and $\tilde{S}^{*}$ and which is also compatible with the $p$-derivations $\delta$ in $S^{*}$ and $\tilde{S}^{*}$. By abuse we shall denote all $\pi_{n}$ by the same letter, $\pi$. Denote by $\operatorname{Prol}_{p}$ the class of all prolongation sequences $S^{*}$ with $S^{n}$ Noetherian, $p$-adically complete, and flat over $\mathbf{Z}_{p}$. Note that, if $S$ is the $p$-adic completion of a smooth $\mathbf{Z}_{p}$-algebra then [6] and [5] 
provide a construction of a prolongation sequence $J^{*}(S) \in \operatorname{Prol}_{p}$ with $J^{0}(S)=S$, such that, for each $n \geqslant 0, J^{n}(S)$ (called the $p$-jet space of $S$ of order $n$ ) is the $p$-adic completion of a smooth $\mathbf{Z}_{p}$-algebra. The prolongation sequence $J^{*}(S)$ has the following universality property: for any prolongation sequence $S^{*}$ with all $S^{n} p$ adically complete, and for any homomorphism $S \rightarrow S^{0}$ there exists a unique morphism of prolongation sequences $J^{*}(S) \rightarrow S^{*}$ prolonging $S \rightarrow S^{0}$.

\section{6. $\delta$-WEIGHTS [2]}

Let $G$ and $G^{\prime}$ be group schemes over $\mathbf{Z}_{p}$. By a $\delta$-homomorphism $\chi: G \rightarrow G^{\prime}$ of order $\leqslant n$ we mean a rule that associates to any prolongation sequence $S^{*} \in \mathbf{P r o l}_{p}$ a group homomorphism

$$
\chi: G\left(S^{0}\right) \rightarrow G^{\prime}\left(S^{n}\right),
$$

which is 'functorial in $S^{*}$ ' in the obvious sense.

Denote by $W$ the ring $\mathbf{Z}[\phi]$ of polynomials with $\mathbf{Z}$-coefficients in an indeterminate $\phi$. (We call $W$ the ring of $\delta$-weights.) If $w=\sum_{i=0}^{n} a_{i} \phi^{i}$ with $a_{i} \in \mathbf{Z}$ and $a_{n} \neq 0$ then we set $\operatorname{deg}(w)=\sum_{i=0}^{n} a_{i}$ and $\operatorname{ord}(w)=n$. There is a natural ring homomorphism

$$
\begin{aligned}
& W \rightarrow\left\{\delta \text {-homomorphisms } \mathbf{G}_{m} \rightarrow \mathbf{G}_{m}\right\}, \quad w \mapsto \chi_{w}, \\
& \chi_{w}(\lambda)=\lambda^{w}:=\lambda^{a_{0}} \phi(\lambda)^{a_{1}} \ldots \phi^{n}(\lambda)^{a_{n}} \in\left(S^{n}\right)^{\times}, \quad \lambda \in\left(S^{0}\right)^{\times} .
\end{aligned}
$$

(Here $\phi^{i}$ are the iterates $i$ times of the map $\phi: S^{j} \rightarrow S^{j+1}, \phi(x)=x^{p}+p \delta x$.)

\subsection{SIEGEL $\delta$-MODULAR FORMS [2]}

Recall that one defines the set $M_{g, m}^{n}$ of Siegel $\delta$-modular functions of genus $g$, order $\leqslant n$, and size $m$ to be the set of all rules $f$ that associate to any prolongation sequence $S^{*} \in \operatorname{Prol}_{p}$ and any triple $(A, \theta, \omega) \in \mathbf{M}_{g}\left(S^{0}\right)$ a matrix

$$
f\left(A, \theta, \omega, S^{*}\right) \in \operatorname{Mat}_{m}\left(S^{n}\right)
$$

satisfying the following properties:

1.7.1. $f\left(A, \theta, \omega, S^{*}\right)$ depends on $S^{*}$ and the isomorphism class of $(A, \theta, \omega)$ only.

1.7.2. The formation of $f\left(A, \theta, \omega, S^{*}\right)$ is functorial in $S^{*}$.

Clearly $M_{g, m}^{n}=\operatorname{Mat}_{m}\left(M_{g, 1}^{n}\right)$.

For our purposes here we review now two special types of Siegel $\delta$-modular forms.

The first type is defined as follows. Let $a, b$ be two distinct nonnegative integers and set $n:=\max \{a, b\}$. Then we define the set $I_{g}\left(\phi^{a}, \phi^{b}\right)$ of isogeny covariant Siegel $\delta$-modular forms of weight $\left(\phi^{a}, \phi^{b}\right)$ as the set of all $f \in M_{g, g}^{n}$ satisfying the following conditions: 
1.7.3. If $(A, \theta, \omega) \in \mathbf{M}_{g}\left(S^{0}\right)$ and $\lambda \in \mathrm{GL}_{g}\left(S^{0}\right)$, then

$$
f\left(A, \theta, \lambda \omega, S^{*}\right)=\phi^{a}(\lambda) \cdot f\left(A, \theta, \omega, S^{*}\right) \cdot \phi^{b}\left(\lambda^{t}\right) .
$$

1.7.4. If $S^{*} \in \operatorname{Prol}_{p},\left(A_{1}, \theta_{1}, \omega_{1}\right),\left(A_{2}, \theta_{2}, \omega_{2}\right) \in \mathbf{M}_{g}\left(S^{0}\right)$, and $u: A_{1} \rightarrow A_{2}$ is any isogeny of degree prime to $p$, such that $u^{*} \omega_{2}=\omega_{1}$, then

$$
f\left(A_{2}, \theta_{2}, \omega_{2}, S^{*}\right)=f\left(A_{1}, \theta_{1}, \omega_{1}, S^{*}\right) \cdot \phi^{b}\left(\left[u^{t}\right]^{t}\right)^{-1} .
$$

By combining the above properties one checks that the following also holds: for any $S^{*} \in \operatorname{Prol}_{p}$, any $\left(A_{1}, \theta_{1}, \omega_{1}\right),\left(A_{2}, \theta_{2}, \omega_{2}\right) \in \mathbf{M}_{g}\left(S^{0}\right)$, and any isogeny $u: A_{1} \rightarrow A_{2}$, of degree prime to $p$,

$$
\phi^{a}\left(\left[u^{t}\right]\right) \cdot f\left(A_{2}, \theta_{2}, \omega_{2}, S^{*}\right)=f\left(A_{1}, \theta_{1}, \omega_{1}, S^{*}\right) \cdot \phi^{b}\left([u]^{t}\right) .
$$

According to [2], Introduction, for each $a, b$ as above $I_{g}\left(\phi^{a}, \phi^{b}\right)$ is a $\mathbf{Z}_{p}$-module of rank one.

The second type of Siegel $\delta$-modular forms we are going to review is the following. Let us fix a $\delta$-weight $w \in W$ of even degree $\operatorname{deg}(w)$ and order $n=\operatorname{ord}(w)$. Define the set $M_{g}(w)$ of Siegel $\delta$-modular forms of weight $w$ as the set of all $f \in M_{g, 1}^{n}$ satisfying the following property:

1.7.5. If $\lambda \in \mathrm{GL}_{g}\left(S^{0}\right)$, then

$$
f\left(A, \theta, \lambda \omega, S^{*}\right)=\chi_{w}(\operatorname{det}(\lambda))^{-1} \cdot f\left(A, \theta, \omega, S^{*}\right) .
$$

Define the set $I_{g}(w)$ of isogeny covariant Siegel $\delta$-modular forms of weight $w$ as the set of all $f \in M_{g}(w)$ satisfying the following property:

1.7.6. If $S^{*} \in \operatorname{Prol}_{p},\left(A_{1}, \theta_{1}, \omega_{1}\right),\left(A_{2}, \theta_{2}, \omega_{2}\right) \in \mathbf{M}_{g}\left(S^{0}\right)$, and $u: A_{1} \rightarrow A_{2}$ is any isogeny of degree prime to $p$, such that $u^{*} \omega_{2}=\omega_{1}$, then

$$
f\left(A_{1}, \theta_{1}, \omega_{1}, S^{*}\right)=d(u)^{-\operatorname{deg}(w) / 2} \cdot f\left(A_{2}, \theta_{2}, \omega_{2}, S^{*}\right) .
$$

Let

$$
\begin{aligned}
& W_{\text {even }}:=\{w \in W \mid \operatorname{deg}(w) \in 2 \mathbf{Z}\}, \\
& W_{-}:=\left\{\sum a_{i} \phi^{i} \in W \mid a_{i} \leqslant 0\right\}, \\
& W_{*}:=W_{-} \cap W_{\text {even }} .
\end{aligned}
$$

Then $W_{*}$ is a $\phi$-stable submonoid of $W_{\text {even }}$ having the property that

$$
\operatorname{ord}\left(w^{\prime}+w^{\prime \prime}\right)=\max \left\{\operatorname{ord}\left(w^{\prime}\right), \operatorname{ord}\left(w^{\prime \prime}\right)\right\}
$$


for all $w^{\prime}, w^{\prime \prime} \in W_{*}$. This property allows us to form a ring $I_{g}$, graded by $W_{*}$ and acted by $\phi$ :

$$
I_{g}:=\bigoplus_{w \in W_{*}} I_{g}(w) \subset M_{g, 1}^{\infty}:=\bigcup_{n} M_{g, 1}^{n} .
$$

The ring $I_{g}$ should be viewed as governing the geometry of the quotient of the moduli space $\mathcal{A}_{g}$ of principally polarized Abelian varieties by the equivalence relation $\sim$ defined by (prime to $p$ degree) isogeny. Indeed, any system of homogeneous elements $f_{0}, \ldots, f_{N} \in I_{g}$ of the same degree gives rise to a (partially defined) map $\mathcal{A}_{g}(R) \rightarrow \mathbf{P}^{N}(R)$ which is constant on (prime to $p$ degree) isogeny equivalence classes; two points of $\mathcal{A}_{g}(R)$ are considered $\sim$-equivalent if there is an isogeny of degree prime to $p$ between the corresponding Abelian schemes over $R$ (where we do not require that the isogeny be compatible with the polarizations). The 'Proj' of $I_{g}$, in an appropriate category of ringed spaces, can then be interpreted as a categorical quotient of $\mathcal{A}_{g}$ by $\sim$. Making the latter precise would require, however, introducing a rather involved formalism; for the sake of clarity and brevity we shall refrain from pursuing this matter in the present paper. Rather, we will prefer to view $I_{g}$ as a substitute for the 'quotient' $\mathcal{A}_{g} / \sim$.

\section{8. $\delta$-MODULAR FORMS ON $D$}

Define the set $M_{D}^{n}$ of $\delta$-modular functions on $D$ to be the set of all rules $f$ that associate to any $S^{*} \in \mathbf{P r o l}_{p}$ and any $(A, i, \theta, \omega) \in \mathbf{M}_{D}\left(S^{0}\right)$ an element $f\left(A, i, \theta, \omega, S^{*}\right) \in S^{n}$ satisfying the following properties:

1.8.1. $f\left(A, i, \theta, \omega, S^{*}\right)$ depends on $S^{*}$ and on the isomorphism class of $(A, i, \theta, \omega)$ only.

1.8.2. The formation of $f\left(A, i, \theta, \omega, S^{*}\right)$ is functorial in $S^{*}$.

Let $w \in W$ be a $\delta$-weight with $\operatorname{deg}(w)$ even and $n:=\operatorname{ord}(w)$. Define the set $M_{D}(w)$ of $\delta$-modular forms of weight $w$ on $D$ to be the set of all $f \in M_{D}^{n}$ satisfying the following property:

1.8.3. If $\lambda \in\left(S^{0}\right)^{\times}$, then

$$
f\left(A, i, \theta, \lambda \omega, S^{*}\right)=\chi_{w}(\lambda)^{-1} \cdot f\left(A, i, \theta, \omega, S^{*}\right) .
$$

Define the set $I_{D}(w)$ of isogeny covariant $\delta$-modular forms of weight $w$ on $D$ to be the set of all $f \in M_{D}(w)$ satisfying the following property: 
1.8.4. If $S^{*} \in \operatorname{Prol}_{p},\left(A_{1}, i_{1}, \theta_{1}, \omega_{1}\right),\left(A_{2}, \theta_{2}, i_{2}, \omega_{2}\right) \in \mathbf{M}_{D}\left(S^{0}\right)$, and $u: A_{1} \rightarrow A_{2}$ is any isogeny of polarized false elliptic curves, of degree prime to $p$, such that $u^{*} \omega_{2}=\omega_{1}$ then

$$
f\left(A_{1}, i_{1}, \theta_{1}, \omega_{1}, S^{*}\right)=\partial(u)^{-\operatorname{deg}(w) / 2} \cdot f\left(A_{2}, i_{2}, \theta_{2}, \omega_{2}, S^{*}\right)
$$

Finally define

$$
I_{D}:=\bigoplus_{w \in W_{*}} I_{D}(w) \subset M_{D}^{\infty}:=\bigcup_{n} M_{D}^{n}
$$

As in [2] the ring $I_{D}$ should be viewed as governing the geometry of the quotient of the Shimura curve (corresponding to $D$ ) by the equivalence relation defined by (prime to $p$ degree) isogeny.

We will prove that for all but finitely many primes $p$ the following holds:

\section{THEOREM 1.2.}

(1) The $\mathbf{Z}_{p}$-modules $I_{D}(w)$ are finitely generated.

(2) $I_{D}(w)=0$ for $\operatorname{deg}(w)>0$.

(3) $I_{D}(0)=\mathbf{Z}_{p}$.

(4) $I_{D}(-1-\phi)$ has rank one.

(5) $I_{D}\left(-1-\phi^{2}\right)$ has rank one.

As in the case of modular curves [1,7], the weights $-1-\phi$ and $-1-\phi^{2}$ in assertions (4) and (5) of the Theorem are, in a sense that will become clear later, the two basic weights in this theory. Assertions (1)-(4) of the Theorem will be proved in the present paper; assertion (5) requires considerably more tools to prove and its proof will be given in a sequel to this paper [9]. For the modular analogues of (4) and (5) we refer to [7] and [1] respectively.

\subsection{INDUCTION MAPS}

The theory in [2] and the theory in the present paper are related via certain induction maps that we now explain. These maps play a key role in our proofs.

PROPOSITION 1.3. There are natural homomorphisms $\operatorname{ind}_{D}: M_{2,1}^{n} \rightarrow M_{D}^{n}$ inducing homomorphisms $\operatorname{ind}_{D}: I_{2}(w) \rightarrow I_{D}(2 w) \quad$ such that if $f \in M_{2,1}^{n}, \quad S^{*} \in$ Prol $_{p}$, $(A, i, \theta, \omega) \in \mathbf{M}_{D}\left(S^{0}\right)$ then

$$
\left(\operatorname{ind}_{D} f\right)\left(A, i, \theta, \omega, S^{*}\right)=f\left(A, \theta, \omega, S^{*}\right)
$$

Proof. Define a rule ind ${ }_{D}$ through the equality in the statement of the Proposition; then it is a trivial exercise to check that if $f \in I_{2}(w)$ then $\operatorname{ind}_{D} f$ belongs indeed to $I_{D}(2 w)$. 
PROPOSITION 1.4. There are natural homomorphisms

$$
\operatorname{ind}_{D}: I_{2}\left(\phi^{a}, \phi^{b}\right) \rightarrow I_{D}\left(-\phi^{a}-\phi^{b}\right)
$$

such that if $f \in I_{2}\left(\phi^{a}, \phi^{b}\right), S^{*} \in \operatorname{Prol}_{p}$, and $(A, i, \theta, \omega) \in \mathbf{M}_{D}\left(S^{0}\right)$, then

$$
f\left(A, \theta, \omega, S^{*}\right)=\left(\operatorname{ind}_{D} f\right)\left(A, i, \theta, \omega, S^{*}\right) \cdot\left[\begin{array}{ll}
1 & 0 \\
0 & d
\end{array}\right] .
$$

Proof. Let $f, S^{*}$, and $(A, i, \theta, \omega)$ be as above.

Claim. The matrix $F:=f\left(A, \theta, \omega, S^{*}\right)$ has the form $c \cdot\left[\begin{array}{ll}1 & 0 \\ 0 & d\end{array}\right]$ for some unique $c \in S^{n}$.

Once we have checked the claim one can define a rule $\operatorname{ind}_{D} f$ by attaching

$$
\left(A, i, \theta, \omega, S^{*}\right) \mapsto c
$$

and it is trivial to check that this rule belongs indeed to $I_{D}\left(-\phi^{a}-\phi^{b}\right)$; this will end the proof.

In order to check the Claim above let us start with any $\alpha \in \mathcal{O}_{D}$ with $\operatorname{det}(\alpha) \not \equiv 0$ $\bmod p$ in $\mathbf{Z}$. Then, by (1.11), $i(\alpha): A \rightarrow A$ is an isogeny of degree prime to $p$. Applying property (1.14) to $f$, and $i(\alpha)$ we get

$$
\left[i(\alpha)^{t}\right] \cdot F=F \cdot[i(\alpha)]^{t} .
$$

Now $[i(\alpha)]=j(\alpha)$ and

$$
\left[i(\alpha)^{t}\right]=\left[i\left(\alpha^{+}\right)\right]=j\left(\alpha^{+}\right)=j(\tau) \cdot j(\alpha) \cdot j(\tau)^{-1} .
$$

So if $G:=j(\tau)^{-1} \cdot F,(1.20)$ becomes

$$
j(\alpha) \cdot G=G \cdot j(\alpha)^{t} .
$$

The above equality holds for all elements $\alpha$ in the set

$$
\mathcal{O}_{D}^{(p)}:=\left\{\alpha \in \mathcal{O}_{D} \mid \operatorname{det}(\alpha) \not \equiv 0 \bmod p\right\} .
$$

Now $\mathcal{O}_{D}^{(p)}$ spans the $\mathbf{Z}$-module $\mathcal{O}_{D}$. (To see this start with some $\alpha \in \mathcal{O}_{D}$ and let $k$ be any integer. We have

$$
\operatorname{det}(k+\alpha)=k^{2}+\operatorname{tr}(\alpha) k+\operatorname{det}(\alpha)
$$

which we may view as an equality in $\mathbf{Z}_{p}$. Since a quadratic equation with coefficients in $\mathbf{F}_{p}$ has at most two solutions in $\mathbf{F}_{p}^{\times}$and since the latter has at least four elements one can find $k \neq \equiv 0 \bmod p$ such that $\operatorname{det}(k+\alpha) \not \equiv 0 \bmod p$. Then we can write 
$\alpha=(k+\alpha)-k$.) We conclude that the equality (1.21) holds for all $\alpha \in \mathcal{O}_{D}$. Since $j\left(\mathcal{O}_{D}\right)$ spans the $\mathbf{Z}_{p}$-module $\operatorname{Mat}_{2}\left(\mathbf{Z}_{p}\right)$ it follows that

$$
H \cdot G=G \cdot H^{t}
$$

for all $H \in \operatorname{Mat}_{2}\left(\mathbf{Z}_{p}\right)$, in particular for

$$
H=\left[\begin{array}{ll}
1 & 0 \\
0 & 0
\end{array}\right] \text { and } H=\left[\begin{array}{ll}
0 & 0 \\
1 & 0
\end{array}\right]
$$

Plugging in these values of $H$ in 1.22 we get that

$$
G=\left[\begin{array}{cc}
0 & -c \\
c & 0
\end{array}\right]
$$

for some $c$ and our Claim is proved.

Remark 1.5. Transposition of matrices defines a homomorphism

$$
I_{2}\left(\phi^{a}, \phi^{b}\right) \rightarrow I_{2}\left(\phi^{b}, \phi^{a}\right), \quad f \mapsto f^{t} .
$$

Then it is trivial to check that $\operatorname{ind}_{D} f=\operatorname{ind}_{D} f^{t}$ for any $f \in I_{2}\left(\phi^{a}, \phi^{b}\right)$.

\subsection{THE RINGS $J_{g}, J_{D}$}

The rings $I_{g}$ and $I_{D}$ are still quite mysterious; only certain graded pieces of these rings have been computed (in [2] and in the present paper). On the other hand, certain natural graded subrings of these rings, that we now introduce, are much better understood. There is a distinguished basis $f^{n}=f_{\text {crys }}^{n}$ of $I_{g}\left(\phi^{n}, \phi^{0}\right)$ constructed with the help of crystalline cohomology; cf. [2]. For the convenience of the reader let us recall that if $S^{*} \in \mathbf{P r o l}_{p}$ and $(A, \theta, \omega) \in \mathbf{M}_{g}\left(S^{0}\right), \omega=\left(\omega_{1}, \ldots, \omega_{g}\right)^{t}$ then $f^{n}\left(A, \theta, \omega, S^{*}\right)$ is the Matrix whose entries are

$$
p^{-1} \cdot\left\langle\phi^{n} \omega_{i}, \omega_{j}\right\rangle_{\theta} \in S^{n},
$$

where we view $\omega_{i}$ as elements of the first deRham module $H$ of $A / S^{0}$, $\phi: H \otimes S^{i} \rightarrow H \otimes S^{i+1}$ are the crystalline liftings of $\phi: S^{i} \rightarrow S^{i+1}$, and $\langle,\rangle_{\theta}$ is the cup product on $H$ defined by $\theta$; we refer to [2] for details. Let $f^{a, b}:=\phi^{b} f^{a-b}$ if $a>b$ and $f^{a, b}:=\left(f^{b, a}\right)^{t}$ if $a<b$. Let

$$
f_{D}^{a}:=\operatorname{ind}_{D} f^{a} \in I_{D}\left(-1-\phi^{a}\right), \quad f_{D}^{a, b}:=\operatorname{ind}_{D} f^{a, b} \in I_{D}\left(-\phi^{a}-\phi^{b}\right) .
$$

So $f_{D}^{a, b}=f_{D}^{b, a}$ and $f_{D}^{a, b}=\phi^{b} f_{D}^{a-b}$ if $a>b$. Let $J_{D} \subset I_{D}$ be the $\mathbf{Z}_{p}$-subalgebra generated by all $f_{D}^{a, b}$. Clearly $J_{D}$ is a graded subalgebra:

$$
J_{D}=\bigoplus_{w \in W_{*}} J_{D}(w)
$$

where $J_{D}(w):=J_{D} \cap I_{D}(w)$.

Recall from [2] that if a sequence of nonnegative integers $a_{1}, \ldots, a_{2 k}$ satisfies $a_{i} \neq a_{i+1}$ for all $1 \leqslant i \leqslant 2 k-1$ and $a_{2 k} \neq a_{1}$ then one can define a Siegel $\delta$-modular 
form (called cyclic product):

$$
\begin{aligned}
f_{a_{1} \ldots a_{2 k}}:= & \operatorname{tr}\left\{f^{a_{1}, a_{2}} \cdot\left(f^{a_{3}, a_{2}}\right)^{*} \cdot f^{a_{3}, a_{4}} \cdot\left(f^{a_{5}, a_{4}}\right)^{*} \cdots f^{a_{2 k-1}, a_{2 k}} \cdot\left(f^{a_{1}, a_{2 k}}\right)^{*}\right\} \\
& \in I_{2}\left(-\phi^{a_{1}}-\cdots-\phi^{a_{2 k}}\right) .
\end{aligned}
$$

Let $J_{g} \subset I_{g}$ be the subalgebra generated by all cyclic products $f_{a_{1} \ldots a_{2 k}}$ and set $J_{g}(w):=J_{g} \cap I_{g}(w)$; then obviously $J_{g}=\bigoplus_{w \in W_{*}} J_{g}(w)$.

Set

$$
f_{a_{1} \ldots a_{2 k}}^{D}:=\operatorname{ind}_{D} f_{a_{1} \ldots a_{2 k}} \in I_{2}\left(-2 \phi^{a_{1}}-\cdots-2 \phi^{a_{2 k}}\right) .
$$

It is trivial to check that

$$
f_{a_{1}, \ldots, a_{2 k}}^{D}=2 \cdot d^{k} f_{D}^{a_{1}, a_{2}} f_{D}^{a_{2}, a_{3}} f_{D}^{a_{3}, a_{4}} \cdots f_{D}^{a_{2 k-1}, a_{2 k}} f_{D}^{a_{2 k}, a_{1}}
$$

so all the $f_{a_{1}, \ldots, a_{2 k}}^{D}$ 's belong to $J_{D}$. In particular we have an induced map

$$
J_{2} \rightarrow J_{D}
$$

This map is, of course, far from being injective; note also that $J_{D}$ is integral over the image of (1.25) because the square of each $f_{D}^{a, b}$ belongs to this image.

On the other hand we will prove that, for all except finitely many primes $p$, the following holds:

THEOREM 1.6. There is a $\phi$-equivariant isomorphism $J_{D} \simeq J_{1}$.

This should be viewed as one more instance of the general principle according to which modular curves and Shimura curves behave in a similar way, in spite of the fact that they are not directly related.

A natural problem is to understand how close the rings $J_{g}$ and $J_{D}$ are to $I_{g}$ and $I_{D}$ respectively. It is tempting to conjecture that the natural maps

$$
J_{g} \otimes_{\mathbf{z}_{p}} \mathbf{Q}_{p} \rightarrow I_{g} \otimes_{\mathbf{z}_{p}} \mathbf{Q}_{p}, \quad J_{D} \otimes_{\mathbf{z}_{p}} \mathbf{Q}_{p} \rightarrow I_{D} \otimes \mathbf{z}_{p} \mathbf{Q}_{p}
$$

are isomorphisms (i.e. all of $I_{g}$ and $I_{D}$ are 'crystalline' over $\mathbf{Q}_{p}$ ). Note on the other hand that $J_{g} \neq I_{g}$ and $J_{D} \neq I_{D}$. Indeed, if $b_{1}:=f_{D}^{1,0} f_{D}^{3,2}$ and $b_{2}:=f_{D}^{2,0} f_{D}^{3,1}$ then the form

$$
\frac{\phi b_{1} \cdot b_{2}^{p}-\phi b_{2} \cdot b_{1}^{p}}{p}
$$

belongs to $I_{D}$ but not to $J_{D}$; a similar example shows that $J_{g} \neq I_{g}$. One can rephrase the above conjecture as follows. Let $J_{g}: p^{\infty}$ be the subring of $I_{g}$ consisting of all elements whose product with a suitable power of $p$ lies in $J_{g}$. Define $J_{D}: p^{\infty}$ similarly. Then the above conjecture says that

$$
J_{g}: p^{\infty}=I_{g}, \quad J_{D}: p^{\infty}=I_{D} .
$$

Let us explain a weaker form of this conjecture (a special case of which we know how to check), which we hope to address in a subsequent paper. Note that the ring $J_{g}: p^{\infty}$ 
is graded and $p$ is a prime element of this ring. So we may consider the ring $\left(J_{g}: p^{\infty}\right)_{((p))}$ of all fractions whose numerator and denominator are homogeneous elements of $J_{g}: p^{\infty}$, of the same degree, with denominator not divisible by $p$. The ring $\left(J_{g}: p^{\infty}\right)_{((p))}$ and its obvious analogues $\left(J_{D}: p^{\infty}\right)_{((p))},\left(I_{g}\right)_{((p))},\left(I_{D}\right)_{((p))}$, turn out to be discrete valuation rings. Then we conjecture that one has isomorphisms between the following $p$-adic completions:

$$
\left(\left(J_{g}: p^{\infty}\right)_{((p)))}\right)^{\wedge} \simeq\left(\left(I_{g}\right)_{((p))}\right)^{\wedge}, \quad\left(\left(J_{D}: p^{\infty}\right)_{((p)))}\right)^{\wedge} \simeq\left(\left(I_{D}\right)_{((p)))}\right)^{\wedge}
$$

(This is of course the same as to have isomorphisms between the residue fields of the above discrete valuation rings.) So far we were able to prove this latter conjecture for $g=1$.

We conclude this Introduction by stating one more result, Theorem 1.7 below, that we obtain as a by-product of our methods. Unlike for the previously stated results we shall only be able, at this point, to state Theorem 1.7 in 'vague terms'; for the precise statement we refer the reader to Theorem 2.3 and the definitions preceding it. What our result below says is that, with a natural definition of SerreTate expansions for Siegel $\delta$-modular forms and with our definition in [2] of Fourier expansions for such forms we have that

THEOREM 1.7. For any form in $J_{g}$ the Serre-Tate and the Fourier expansions 'formally coincide', provided $g \leqslant 3$.

We expect that an appropriate generalization of this result holds for arbitrary $g$.

\section{Serre-Tate and Fourier Expansions}

\subsection{SERRE-TATE EXPANSION OF SIEGEL $\delta$-MODULAR FORMS}

Let $A_{0}$ be an ordinary Abelian variety over $k:=\overline{\mathbf{F}}_{p}$. The universal formal deformation space of $A_{0}$, [19], p. 149, 159, (parameterizing liftings of $A_{0}$ to local Artin rings with residue field $k$ ) is canonically isomorphic as a functor to

$$
\operatorname{Hom}_{\mathbf{Z}_{p}}\left(T_{p}\left(A_{0}\right) \otimes T_{p}\left(\check{A}_{0}\right), \mathbf{G}_{m}^{\text {for }}\right),
$$

where $T_{p}\left(A_{0}\right)$ and $T_{p}\left(\check{A}_{0}\right)$ are the 'physical' Tate modules of $A_{0}$ and its dual $\check{A}_{0}$ i.e. the inverse limits of the $p$-power torsion $k$-points of $A_{0}$ and $\check{A}_{0}$, respectively. For any formal Abelian scheme $A$ over a complete local ring $S$ with residue field $k$ which lifts $A_{0}$ we denote by

$$
q(A / S ;-,-): T_{p}\left(A_{0}\right) \times T_{p}\left(\check{A}_{0}\right) \rightarrow \mathbf{G}_{m}^{\mathrm{for}}(S)=1+m_{S}
$$

the corresponding bilinear map, where $m_{S}$ is the maximal ideal of $S$. In particular, if we are given bases $b, \breve{b}$ of $T_{p}\left(A_{0}\right)$ and $T_{p}\left(\breve{A}_{0}\right)$ then

$$
q_{i j}:=q\left(A / S ; b_{i}, \check{b}_{j}\right) \in 1+m_{S}
$$


are called the Serre-Tate parameters of $A / S ; q=\left(q_{i j}\right)$ is called the Serre-Tate matrix. If $R=\hat{\mathbf{Z}}_{p}^{u r}$ is the completion of the maximum unramified extension of $\mathbf{Z}_{p}$ then the universal formal deformation space can be identified, via $b, \breve{b}$, with $\operatorname{Spf} R\left[\left[t_{i j}\right]\right]$; any $A / S$ as above corresponds to the map

$$
\left.R\left[t_{i j}\right]\right] \rightarrow S, \quad t_{i j} \mapsto q_{i j}-1 .
$$

Finally let us recall from [19], p. 161, that there is a functorial isomorphism (viewed sometimes later as an identification)

$$
T_{p}\left(\check{A}_{0}\right) \simeq \operatorname{Hom}_{S}\left(A^{\text {for }}, \mathbf{G}_{m}^{\text {for }}\right),
$$

hence any basis $\check{b}$ of $T_{p}\left(\check{A}_{0}\right)$ induces, by pull-back of the standard invariant 1-form $\mathrm{d} T / T$ on $\mathbf{G}_{m}^{\text {for }}$, a basis $\omega=\omega(\check{b})$ of 1-forms on $A / S$ :

$$
\omega=\check{b}^{*} \frac{\mathrm{d} T}{T},
$$

where, as a rule we see $\omega$ and $\check{b}$ as column vectors.

It is convenient to make the following definition: by a $g$-frame we will understand a tuple

$$
\left(A_{0}, \theta_{0}, b, \check{b}\right)
$$

where $\left(A_{0}, \theta_{0}\right)$ is a principally polarized ordinary Abelian variety of dimension $g$ over $k$ and $b, \breve{b}$ are bases of the Tate modules $T_{p}\left(A_{0}\right)$ and $T_{p}\left(\breve{A}_{0}\right)$ which correspond to each other via $\theta_{0}$. Let us fix, in what follows, a $g$-frame as above.

Let $R\left[\left[t_{i j}\right]\right], 1 \leqslant i, j \leqslant g$ be the universal formal deformation ring of $A_{0}$ corresponding to the bases $b, b$. By the symmetry property in [19], p. 149,

$$
S_{\mathrm{def}}:=R\left[\left[t_{i j}\right]\right] /\left(t_{i j}-t_{j i}\right)
$$

is the universal formal deformation ring for $\left(A_{0}, \theta_{0}\right)$ (Spf $S_{\text {def }}$ parameterizes liftings of $\left(A_{0}, \theta\right)$ to Artin local algebras with residue field $k$ ). The universal formal Abelian scheme $A_{\text {def }} / S_{\text {def }}$ has a principal polarization $\theta_{\text {def }}$ hence it is an Abelian scheme. We need to review a few basic facts from crystalline theory; cf. [14], 1.4.2., 2.1.3., [19] p. 175, [16], p. 116. Let $H$ be the first deRham module of $A_{\text {def }} / S_{\text {def }}$. Then $H$ comes equipped with its Hodge filtration and with the Gauss-Manin connection $\nabla$. Moreover, for any lifting of Frobenius $\left.\phi: R\left[t_{i j}\right]\right] \rightarrow R\left[\left[t_{i j}\right]\right]$ there is a $\phi$-linear endomorphism (still denoted by) $\phi: H \rightarrow H$ induced by the crystalline nature of $H$. Then one can canonically complete $\omega=\omega(\check{b})$ to a basis $\omega, u$ of $H$, compatible with the Hodge filtration, such that $\phi u=u$ for any lifting of Frobenius $\phi: R\left[\left[t_{i j}\right]\right] \rightarrow R\left[\left[t_{i j}\right]\right]$ and such that

$$
\nabla u=0, \quad \nabla \omega=(\mathrm{d} \log q) \otimes u,
$$

where $q=\left(q_{i j}\right), q_{i j}:=1+t_{i j}$. The basis $(\omega, u)$ is canonically associated to the data $A_{0}, b, \breve{b}$ (and actually does not depend on $\theta_{0}$ ). Let

$$
\omega_{\text {def }}, u_{\text {def }} \in H \otimes S_{\text {def }}
$$


be the images of $\omega, u$. (We will sometimes denote these images simply by $\omega, u$.) Then

$$
\left(A_{\text {def }}, \theta_{\text {def }}, \omega_{\text {def }}\right) \in \mathbf{M}_{g}\left(S_{\text {def }}\right) .
$$

On the other hand, one can consider the prolongation sequence $S_{\mathrm{def}}^{*}$ defined by

$$
S_{\text {def }}^{n}:=R\left[\left[t_{i j}\right]\right]\left[t_{i j}^{\prime}, \ldots, t_{i j}^{(n)}\right]^{\wedge} /\left(t_{i j}-t_{j i}, t_{i j}^{\prime}-t_{j i}^{\prime}, \ldots, t_{i j}^{(n)}-t_{j i}^{(n)}\right),
$$

where $\delta t_{i j}^{(s)}=t_{i j}^{(s+1)}$. Set

$$
S_{\mathrm{def}}^{\infty}:=\bigcup_{n} S_{\mathrm{def}}^{n}
$$

We may define, then, the Serre-Tate expansion map:

$$
E_{A_{0}}: M_{g, 1}^{\infty}=\bigcup_{n} M_{g, 1}^{n} \rightarrow S_{\mathrm{def}}^{\infty}
$$

by the formula

$$
E_{A_{0}}(f):=f\left(A_{\mathrm{def}}, \theta_{\mathrm{def}}, \omega_{\mathrm{def}}, S_{\mathrm{def}}^{*}\right) .
$$

The map (2.8) is canonically attached to the $g$-frame (2.4) but, by abuse of notation, we only indicate its dependence on $A_{0}$.

It will be useful to consider a certain subring and a certain overing of $S_{\text {def }}^{\infty}$ as follows. Let $\mu:=g(g+1) / 2$ and let $\phi^{*} \Psi_{* *}$ be a collection of variables $\phi^{r} \Psi_{i j}$, $r \geqslant 0,1 \leqslant i, j \leqslant g$, such that $\phi^{r} \Psi_{i j}=\phi^{r} \Psi_{j i}$. We may consider the injective ring homomorphisms:

$$
\mathbf{Z}_{p}\left[\phi^{*} \Psi_{* *}\right] \rightarrow S_{\text {def }}^{\infty} \rightarrow \operatorname{Maps}\left((1+p R)^{\mu}, R\right)
$$

defined as follows. The first sends

$$
\phi^{r} \Psi_{i j} \mapsto \frac{1}{p} \phi^{r} \log \frac{\phi\left(1+t_{i j}\right)}{\left(1+t_{i j}\right)^{p}}=\frac{1}{p} \phi^{r} \log \left(1+p \frac{t_{i j}^{\prime}+\frac{\left.1+t_{i j}^{p}-\left(1+t_{i j}\right)^{p}\right)}{p}}{\left(1+t_{i j}\right)^{p}}\right),
$$

where $\phi$ acts on $S_{\text {def }}^{\infty}$ by

$$
\phi\left(t_{i j}^{(r)}\right):=\left(t_{i j}^{(r)}\right)^{p}+t_{i j}^{(r+1)} .
$$

The second map in (2.10) sends

$$
t^{(r)} \mapsto\left(q \mapsto \delta^{r}(q-1)\right),
$$

where $q$ is viewed as a symmetric matrix $\left(q_{i j}\right)$ with $R$-coefficients and $\delta: R \rightarrow R$ is given by $\delta x=\left(\phi(x)-x^{p}\right) / p$ where $\phi: R \rightarrow R$ is the unique lifting of Frobenius. So the composition (2.10) sends

$$
\phi^{r} \Psi_{i j} \mapsto\left(q \mapsto\left(\phi^{r} \Psi_{i j}\right)(q):=\frac{1}{p} \phi^{r} \log \frac{\phi\left(q_{i j}\right)}{q_{i j}^{p}}\right) .
$$

From now on we regard (2.10) as an inclusion. 
The following result is an analogue of the Fourier expansion principle proved in [2], Theorem 3.1. We shall refer to the Theorem below as the Serre-Tate expansion principle.

THEOREM 2.1. (1) The restriction of the Serre-Tate expansion map $E_{A_{0}}: M_{g, 1}^{\infty} \rightarrow S_{\mathrm{def}}^{\infty}$ to any space $M_{g}(w)$ is injective with torsion free cokernel.

(2) The induced maps $M_{g}(w) \otimes_{\mathbf{z}_{p}} R \rightarrow S_{\text {def }}^{\infty}$ are injective.

Proof. (sketch) The proof of assertion 1 is entirely similar to the (rather lengthy) proof of [2], Theorem 3.1, and shall be omitted. We shall give a brief sketch of the proof of assertion (2). Let us fix a weight $w$ of order $n$ and let us define Siegel modular $\delta$-forms over $R$ of weight $w$ by using exactly the same definition as for Siegel modular $\delta$-forms of weight $w$ except that we are asking now that all prolongation sequences involved be over $R$. Denote by $M_{g, R}(w)$ the $R$-module of all Siegel modular $\delta$-forms over $R$ of weight $w$. Then the proof of Theorem 3.1 in [2] extends, without modifications, to provide an injective map

$$
M_{g, R}(w) \rightarrow S_{\text {def }}^{\infty}
$$

We shall be done if we prove that the natural map

$$
M_{g}(w) \otimes \mathbf{Z}_{p} R \rightarrow M_{g, R}(w)
$$

is injective. This follows from the following Lemma applied to the ring $A$ of global functions on the $n$th $p$-jet space of an affine open set of the moduli space of principally polarized Abelian schemes with level $N \geqslant 3$ structure over $\mathbf{Z}_{p}$. We leave details to the reader; what one needs to do is use the setting of the proof of [2], Theorem 3.1, which links Siegel $\delta$-modular forms to $p$-jet spaces of moduli spaces.

LEMMA 2.2. Let $A$ be a smooth $\mathbf{Z}_{p}$-algebra and, as usual, $R=\hat{\mathbf{Z}}_{p}^{u \text {. }}$. Assume Spec A/pA is geometrically connected. Then the map

$$
\hat{A} \otimes \mathbf{Z}_{p} R \rightarrow\left(A \otimes_{\mathbf{Z}_{p}} R\right)^{\wedge}
$$

is injective.

As usual, the ^ sign denoted $p$-adic completion.

Proof. Let $R_{1}$ be a finite extension of $\mathbf{Z}_{p}$ contained in $R$ such that if

$$
A_{1}:=A \otimes_{\mathbf{Z}_{p}} R_{1}, \quad k_{1}:=R_{1} / p R_{1},
$$

then Spec $A_{1} / p A_{1}$ has a $k_{1}$-point. This point lifts, by smoothness, to an $R_{1}$-point of Spec $A_{1}$, given, say, by $\pi: A_{1} \rightarrow R_{1}$. One can find an etale map Spec $A_{1} \rightarrow$ Spec $R_{1}[T]$ (where $T$ is a tuple of indeterminates) such that $\pi$ is mapped to the point $T \mapsto 0$. Let $P=\operatorname{Ker}(\pi)$. Then the completion of the local ring of $A_{1}$ at $P$ is 
isomorphic to $R_{1}[[T]]$. We have induced ring homomorphisms

$$
R_{1}[T] \stackrel{u}{\rightarrow} \hat{A}_{1} \stackrel{v}{\rightarrow} R_{1}[[T]]
$$

We claim that $v$ above is injective. Indeed, since $\hat{A}_{1}$ is $p$-adically separated, it is enough to show that the induced map

$$
\bar{v}: \hat{A}_{1} / p \hat{A}_{1}=A_{1} / p A_{1} \rightarrow k_{1}[[T]]
$$

is injective. Assume the prime ideal $\operatorname{Ker}(\bar{v})$ is not zero and seek a contradiction. Note that $A_{1} / p A_{1}$ is an integral domain (by smoothness of $A$ and geometric connectivity of $A / p A$ ). On the other hand, $\operatorname{Ker}(\bar{v}) \cap k_{1}[T]=0$ because $k_{1}[T] \rightarrow k_{1}[[T]]$ is injective. It follows that $\operatorname{Spec} A_{1} / p A_{1} \rightarrow \operatorname{Spec} k_{1}[T]$ is not quasi-finite, a contradiction. So our claim that $v$ is injective is proved. Let $K_{1}=R_{1}[1 / p], K=R[1 / p]$. Since $R_{1}$ is finite over $\mathbf{Z}_{p}$ we have

$$
\hat{A} \otimes \mathbf{Z}_{p} R \simeq \hat{A}_{1} \otimes_{R_{1}} R
$$

On the other hand, we have a commutative diagram

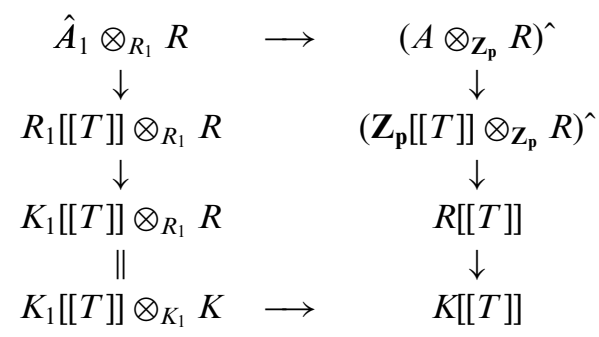

Since $R$ is flat over $R_{1}$ the composition of the left vertical arrows is injective. So it is enough to check that the bottom horizontal arrow is injective. This is, however, trivial.

\subsection{THE CASE $g \leqslant 3$}

Let us say that a $g$-frame $\left(A_{0}, \theta_{0}, b, \check{b}\right)$ (cf. (2.9)) is of Jacobian type if $\left(A_{0}, \theta_{0}\right)=$ $\left(\mathrm{Jac}\left(C_{0}\right), \theta_{C_{0}}\right)$ for some curve $C_{0}$ of genus $g \in\{1,2,3\}$ and, in case $g=3, C_{0}$ is nonhyperelliptic. Of course $\theta_{C_{0}}$ above denotes the theta polarization on $\operatorname{Jac}\left(C_{0}\right)$. The 'Jacobian type' hypothesis will allow us to compute the Serre-Tate expansion map more explicitly.

Let Spf $R\left[\left[s_{1}, \ldots, s_{\mu}\right]\right]$ be the universal deformation space of $C_{0}$. By the symmetry property in [19], p. 149, the map $R\left[\left[t_{i j}\right]\right] \rightarrow R\left[\left[s_{1}, \ldots, s_{\mu}\right]\right]$ factors through $R\left[\left[t_{i j}\right]\right] /\left(t_{i j}-t_{j i}\right)$. By [27], p. 167, the reduction $\bmod p$ of the map

$$
S_{d e f}=R\left[\left[t_{i j}\right]\right] /\left(t_{i j}-t_{j i}\right) \rightarrow R\left[\left[s_{1}, \ldots, s_{\mu}\right]\right]
$$


is surjective, hence an isomorphism. It follows that the map (2.11) itself is an isomorphism. (We may, and will, assume that $s_{1}, \ldots, s_{\mu}$ are the classes of $t_{i j}$ for $i \leqslant j$.) Note that on $H \otimes S_{\text {def }}$ we have available the cup product $\langle-,-\rangle$ coming from the universal curve. By the compatibility of the cup product with the Gauss-Manin connection we get $\nabla\left\langle\omega, u^{t}\right\rangle=0$ (here $\omega, u$ are viewed as column vectors and the upper $t$ means 'transposition') so the entries of the matrix $\left\langle\omega, u^{t}\right\rangle$ belong to $R$. On the other hand one can consider the lifting of Frobenius $\phi$ on $S_{\text {def }}=R\left[\left[t_{i j}\right]\right] /\left(t_{i j}-t_{j i}\right)$ induced by $\phi\left(q_{i j}\right)=q_{i j}^{p}$, where $q_{i j}=1+t_{i j}$; by [14], 2.1.3., we have, for this $\phi$, that $\phi \omega=p \omega$. Using the equality $\left\langle\phi \omega, \phi u^{t}\right\rangle=p \cdot \phi\left\langle\omega, u^{t}\right\rangle$ we get that $\epsilon:=-\left\langle\omega, u^{t}\right\rangle$ has the property that $\phi \epsilon=\epsilon$. Hence $\epsilon \in \mathrm{GL}_{2}\left(\mathbf{Z}_{p}\right)$. Let $C / R$ be any curve lifting $C_{0} / k$; such a curve corresponds to a matrix $t(C)=\left(t_{i j}(C)\right)$ with entries in $p R$. We set $q(C):=1+t(C)$. For each such $C$ we obtain a basis $\omega(C), u(C)$ of $H(C):=H_{\mathrm{DR}}^{1}(C / R)=H_{\mathrm{DR}}^{1}(\operatorname{Jac}(C) / R)$. This basis is compatible with the Hodge filtration. We claim that $u(C)$ is fixed by the crystalline $\phi: H(C) \rightarrow H(C)$ induced by the unique lifting of Frobenius $\phi: R \rightarrow R$; i.e. $\phi u(C)=u(C)$. Indeed one can easily construct a lifting of Frobenius $\phi$ on $R\left[\left[t_{i j}\right]\right]$ such that the homomorphism

$$
R\left[\left[t_{i j}\right]\right] \rightarrow R, \quad t \mapsto t(C)
$$

commutes with the action of $\phi$ on the two rings: just let

$$
\phi t=t^{p}+\left\{\phi(t(C))-t(C)^{p}\right\} .
$$

In particular the induced homomorphism $H \rightarrow H(C)$ is $\phi$-equivariant. Now one uses the fact that $u$ is fixed by any lifting of Frobenius, in particular by $\phi: H \rightarrow H$; it follows that $u(C)$ is fixed by $\phi: H(C) \rightarrow H(C)$ and our claim is proved. By [18], pp. 190-191, we have $\left\langle u(C), u(C)^{t}\right\rangle=0$. As in loc. cit. we can consider, for each $C$, a column vector $\beta(C)$ of elements of $H(C)$ such that

$$
\begin{aligned}
& \phi \beta(C)=p \cdot \beta(C), \\
& \left\langle u(C), \beta(C)^{t}\right\rangle=I, \\
& \left\langle\beta(C), u(C)^{t}\right\rangle=-I, \\
& \left\langle\beta(C), \beta(C)^{t}\right\rangle=0 .
\end{aligned}
$$

Express

$$
\omega(C)=\Omega_{1}(C) \cdot \beta(C)+\Omega_{2}(C) \cdot u(C),
$$

where $\Omega_{i}(C)$ are matrices with entries in $R$. We get that

$$
\Omega_{1}(C)=\epsilon .
$$

Also, by [16], p. 116, we have

$$
\Omega_{2}(C)=\log (q(C)) .
$$


Finally, from the equality $\left\langle\omega(C), \omega(C)^{t}\right\rangle=0$ we get

$$
0=\epsilon \cdot \log (q(C))-\log (q(C)) \cdot \epsilon^{t} .
$$

Since this is true for any $C$ it follows that $\epsilon$ must be a scalar matrix:

$$
\epsilon \in \mathbf{Z}_{p}^{\times} \cdot I
$$

We conclude that

$$
\omega(C)=\epsilon \cdot \beta(C)+\log (q(C)) \cdot u(C)
$$

If $g=1$ one can choose $\epsilon=1$ by [14], Introduction.

The composition of the Serre-Tate expansion map (2.8)

$$
E_{A_{0}}: M_{g, 1}^{\infty} \rightarrow S_{\mathrm{def}}^{\infty}
$$

with the embedding

$$
S_{\text {def }}^{\infty} \rightarrow \operatorname{Maps}\left((1+p R)^{\mu}, R\right)
$$

is given by the map

$$
M_{g, 1}^{\infty} \rightarrow \operatorname{Maps}\left((1+p R)^{\mu}, R\right)
$$

described as follows. Let $f \in M_{g, 1}^{n}$ and let $q \in(1+p R)^{\mu}$. We identify $q$, as usual, with a symmetric matrix $\left(q_{i j}\right)$ with entries in $1+p R$. Let $C / R$ be the unique lifting of $C_{0} / k$ such that $q(C)=q$. Then

$$
E_{A_{0}}(f)(q)=f\left(\operatorname{Jac}(C), \theta_{C}, \omega(C), R^{*}\right) \in R,
$$

where $\theta_{C}$ is the theta divisor on $\operatorname{Jac}(C)$ and $R^{*}$ is the unique prolongation sequence all of whose rings are $R$.

\subsection{COMPARISON BETWEEN SERRE-TATE AND FOURIER EXPANSIONS}

Recall that we defined in [2] a Fourier expansion map

$$
E_{\infty}: M_{g, 1}^{\infty} \rightarrow S_{\text {can }}^{\infty}:=\bigcup_{n} \mathbf{Z}_{p}\left[\left[q_{*}\right]\right]\left[q_{*}^{-1}\right]\left[q_{*}^{\prime}, \ldots, q_{*}^{(n)}\right]^{\wedge}
$$

where $q_{*}, q_{*}^{\prime}, \ldots, q_{*}^{(n)}$ are $\mu$-vectors with entries variables. Note that $\phi$ acts on $S_{\text {can }}^{\infty}$ by 
the formula

$$
\phi\left(q_{*}^{(i)}\right)=\left(q_{*}^{(i)}\right)^{p}+p q_{*}^{(i+1)}
$$

and 2.16 is $\phi$-equivariant.

(Here is a parenthetical remark. The $q$ 's in (2.15) and the $q_{*}$ 's in (2.16) are unrelated; the former ones are the 'Serre-Tate parameters' while the latter are the 'Fourier variables'. In using the same letter $q$ in both cases we are following an established, though somewhat misleading, convention.)

There is an embedding

$$
\begin{aligned}
& \mathbf{Z}_{p}\left[\phi^{*} \Psi_{* *}\right] \rightarrow S_{\mathrm{can}}^{\infty}, \\
& \phi^{r} \Psi_{i j} \mapsto \frac{1}{p} \phi^{r} \log \frac{\phi\left(q_{i j}\right)}{q_{i j}^{p}} .
\end{aligned}
$$

By [2]

$$
E_{\infty}\left(J_{g}\right) \subset \mathbf{Z}_{p}\left[\phi^{*} \Psi_{* *}\right] .
$$

THEOREM 2.3. Assume the $g$-frame $\left(A_{0}, \theta_{0}, b, \check{b}\right)$ is of Jacobian type. Then

$$
E_{A_{0}}\left(J_{g}\right) \subset \mathbf{Z}_{p}\left[\phi^{*} \Psi_{* *}\right]
$$

and, for any $f \in J_{g}(w)$ we have

$$
E_{A_{0}}(f)=\epsilon^{\frac{\operatorname{deg}(w)}{2} g} \cdot E_{\infty}(f)
$$

in $\mathbf{Z}_{p}\left[\phi^{*} \Psi_{* *}\right]$.

Here $\epsilon \in \mathbf{Z}_{p}^{\times}$is the unit introduced in the previous section. Note that the Theorem should be viewed as somewhat surprising for, as noted before, there is no direct relation between the Serre-Tate parameters and the Fourier variables.

Proof. We may assume $f$ is a cyclic product. Then the required equality follows from the Lemma below.

LEMMA 2.4. Assume the g-frame $\left(A_{0}, \theta_{0}, b, \check{b}\right)$ is of Jacobian type and let $f_{i j}^{n} \in M_{g, 1}^{n}$ denote the $(i, j)$-component of the matrix $f^{n}=f_{\text {crys }}^{n} \in \operatorname{Mat}_{g}\left(M_{g, 1}^{n}\right)$. Then the equality $E_{A_{0}}\left(f_{i j}^{n}\right)=\epsilon \cdot E_{\infty}\left(f_{i j}^{n}\right)$ holds in the ring $\mathbf{Z}_{p}\left[\phi^{*} \Psi_{* *}\right]$.

Proof. By [2], Section 4, we know that

$$
E_{\infty}\left(f_{i j}^{n}\right)=\phi^{n-1} \Psi_{i j}+p \phi^{n-2} \Psi_{i j}+\cdots+p^{n-1} \Psi_{i j} .
$$


On the other hand, to compute $E_{A_{0}}(f)$ take any $q \in(1+p R)^{\mu}$ and take a curve $C / R$ lifting $C_{0} / k$ with $q(C)=q$; using the definition of $f_{\text {crys }}^{n}$ in [2], Section 4, and using formulas (2.13) and (2.15) we get

$$
\begin{aligned}
E_{A_{0}}\left(f^{n}\right)(q) & =f^{n}\left(\operatorname{Jac}(C), \theta_{C}, \omega(C), R^{*}\right)=\frac{1}{p}\left\langle\phi^{n} \omega(C), \omega(C)^{t}\right\rangle \\
& =\frac{1}{p}\left\langle\epsilon \cdot p^{n} \cdot \beta(C)+\left(\log \phi^{n}(q)\right) \cdot u(C), \epsilon \cdot \beta(C)^{t}+u(C)^{t} \cdot(\log q)\right\rangle \\
& =\frac{\epsilon}{p}\left\{\log \phi^{n}(q)-p^{n} \cdot \log q\right\} \\
& =\frac{\epsilon}{p}\left\{\left[\log \phi^{n}(q)-p \cdot \log \phi^{n-1}(q)\right]+\cdots+\left[p^{n-1} \cdot \log \phi(q)-p^{n} \cdot \log q\right\}\right. \\
& =\epsilon\left\{\phi^{n-1} \Psi+\cdots+p^{n-1} \Psi\right\}(q),
\end{aligned}
$$

which ends the proof.

\subsection{SERRE-TATE EXPANSION OF $\delta$-MODULAR FORMS ON $D$}

Our aim in what follows is to define Serre-Tate expansions for elements of $M_{D}^{n}$. (Of course, there is no reasonable 'Fourier expansion' for such forms because Shimura curves associated to $D$ are proper.) We need to discuss some preliminaries. Let $\left(A_{0}, i_{0}, \theta_{0}\right)$ be a polarized false elliptic curve over $k:=\overline{\mathbf{F}}_{p}$, with $A_{0}$ ordinary. Let us denote by $S_{\mathrm{def}, D}=R[[T]]$ the universal formal deformation ring of $\left(A_{0}, i_{0}\right)$; so $S p f S_{\text {def }, D}$ parameterizes liftings of $\left(A_{0}, i_{0}\right)$ to false elliptic curves over local Artin rings with residue field $k$ (cf. [12]). Also let $A_{\mathrm{def}, D} / S_{\mathrm{def}, D}$ be the corresponding universal formal Abelian scheme. Note that $A_{\text {def, } D}$ has a natural structure of polarized false elliptic curve: $\left(A_{\mathrm{def}, D}, i_{\mathrm{def}, D}, \theta_{\mathrm{def}, D}\right)$. Indeed the reductions mod $p^{n}$ of $A_{\mathrm{def}, D}$ have an $\mathcal{O}_{D}$-action and a unique principal polarization such that the Rosati involution on $\operatorname{End}\left(A_{0}\right)$ induces the + involution on $\mathcal{O}_{D}$. Hence the formal scheme $A_{\text {def }, D}$ has an $\mathcal{O}_{D}$-action and a principal polarization such that the Rosati involution on End $\left(A_{0}\right)$ induces the + involution on $\mathcal{O}_{D}$. By Grothendieck's existence theorem $A_{\text {def }, D}$ is algebraisable, hence it 'is' an Abelian scheme. Since $\operatorname{End}\left(A_{\mathrm{def}, D}\right) \rightarrow$ $\operatorname{End}\left(A_{0}\right)$ is injective the Rosati involution on $\operatorname{End}\left(A_{\text {def }, D}\right)$ induces the + involution on $\mathcal{O}_{D}$. This proves that $A_{\text {def }, D}$ 'is' a polarized false elliptic curve. Note that if one chooses bases $b, \breve{b}$ of $T_{p}\left(A_{0}\right), T_{p}\left(\check{A_{0}}\right)$ that correspond to each other via $\theta_{0}$ then one gets an induced ring homomorphism

$$
\rho: S_{\text {def }}=R\left[\left[t_{i j}\right]\right] /\left(t_{12}-t_{21}\right) \rightarrow S_{\text {def }, D}=R[[T]] .
$$

The following Lemma will help us choose the bases $b, \check{b}$ wisely:

LEMMA 2.5. Let $\left(A_{0}, i_{0}, \theta_{0}\right)$ be a polarized false elliptic curve over $k=\overline{\mathbf{F}}_{p}$ such that $A_{0}$ is ordinary. Then there exist $\mathbf{Z}_{p}$-bases $b$ and $\breve{b}$ of the 'physical' Tate modules $T_{p}\left(A_{0}\right)$ 
and $T_{p}\left(\check{A}_{0}\right)$ respectively, corresponding to each other via $\theta_{0}$, such that for any false elliptic curve $(A, i)$ over $R$ equipped with an isomorphism $(A, i) \otimes \overline{\mathbf{F}}_{p} \simeq\left(A_{0}, i_{0}\right)$ the Serre-Tate matrix of $(A, i)$ (with respect to $b, \breve{b}$ ) has the form

$$
\left[\begin{array}{cc}
q & 0 \\
0 & q^{d}
\end{array}\right]
$$

where $q \in 1+p R$. In particular, with respect to the bases $b, \breve{b}$, the homomorphism $\rho$ in (2.19) sends

$$
t_{11} \mapsto T, \quad t_{22} \mapsto(T+1)^{d}-1, \quad t_{12}, t_{21} \mapsto 0 .
$$

Furthermore, viewing $b$ as a column vector, we have:

$$
i(\alpha) b=j(\alpha) b, \quad \alpha \in \mathcal{O}_{D} .
$$

In particular, the above Lemma gives an explicit 'formal linearization' of the corresponding Shimura curve. Of course this is just an instance (made explicit and more precise, for our purposes) of the general linearization results of Noot [26] and Moonen [24].

Proof. Let $E_{\mathrm{def}} / R[[T]]$ be the universal formal deformations of $E_{0} / k$, where $E_{0}$ is any ordinary elliptic curve, corresponding to the choice of bases $v, \check{v}$ of $T_{p}\left(E_{0}\right)$ and $T_{p}\left(\check{E}_{0}\right)$. (We do not assume $v, \breve{v}$ correspond to each other under the canonical polarization on $E_{0}$. Also, we do not fix $v, \breve{v}$ yet; we shall fix them later, in the course of our proof.) By [12] we have an isomorphism of $p$-divisible groups

$$
A_{\text {def }, D}\left[p^{\infty}\right] \simeq E_{\text {def }}\left[p^{\infty}\right] \times E_{\text {def }}\left[p^{\infty}\right]
$$

over $R[[T]]$ which is $\mathcal{O}_{D}$-equivariant. Now the lifting $(A, i)$ of $\left(A_{0}, i_{0}\right)$ gives rise to a ring homomorphism

$$
R[[T]] \rightarrow R, \quad T \mapsto q-1 .
$$

Let $E$ be the pull-back of $E_{\text {def }}$ via (2.22). By Grothendieck's existence theorem $E_{\text {def }}, E$ are actually elliptic curves (rather than 'formal elliptic curves'). Now we have an induced isomorphism of $p$-divisible groups

$$
A\left[p^{\infty}\right] \simeq E\left[p^{\infty}\right] \times E\left[p^{\infty}\right]
$$

and corresponding isomorphisms between the connected components of (2.23) reduced $\bmod p$ :

$$
A_{0}^{\text {for }} \simeq E_{0}^{\text {for }} \times E_{0}^{\text {for }}
$$


and the etale quotients of (2.23) reduced $\bmod p$ :

$$
T_{p}\left(A_{0}\right) \otimes_{\mathbf{Z}_{p}}\left(\mathbf{Q}_{p} / \mathbf{Z}_{p}\right) \simeq\left(T_{p}\left(E_{0}\right) \otimes_{\mathbf{Z}_{p}}\left(\mathbf{Q}_{p} / \mathbf{Z}_{p}\right)\right) \times\left(T_{p}\left(E_{0}\right) \otimes_{\mathbf{z}_{p}}\left(\mathbf{Q}_{p} / \mathbf{Z}_{p}\right)\right) .
$$

Taking Hom $\left(-, \mathbf{G}_{m}^{\text {for }}\right)$ in 2.24 and taking the functor 'Tate module' in (2.25) we get isomorphisms

$$
\begin{aligned}
& T_{p}\left(\check{A}_{0}\right) \simeq T_{p}\left(\check{E}_{0}\right) \oplus T_{p}\left(\check{E}_{0}\right), \\
& T_{p}\left(A_{0}\right) \simeq T_{p}\left(E_{0}\right) \oplus T_{p}\left(E_{0}\right) .
\end{aligned}
$$

View the elements of the right hand sides of (2.26) and (2.27) as column vectors; then (2.27) is $\mathrm{Mat}_{2}\left(\mathbf{Z}_{p}\right)$-equivariant whereas (2.26) is $\mathrm{Mat}_{2}\left(\mathbf{Z}_{p}\right)^{o p}$-equivariant with respect to the action on $T_{p}\left(A_{0}\right)$ induced by

$$
\begin{aligned}
& \mathcal{O}_{D}^{\text {op }} \rightarrow \operatorname{End}\left(\check{A}_{0} / k\right) \\
& \alpha \mapsto i(\alpha)^{\llcorner}
\end{aligned}
$$

and the action

$$
\operatorname{Mat}_{2}\left(\mathbf{Z}_{p}\right)^{\text {op }} \times\left(T_{p}\left(\check{E}_{0}\right) \oplus T_{p}\left(\check{E}_{0}\right)\right) \rightarrow T_{p}\left(\check{E}_{0}\right) \oplus T_{p}\left(\check{E}_{0}\right)
$$

given by

$$
\left(X,\left[\begin{array}{c}
\breve{w}_{1} \\
\check{w}_{2}
\end{array}\right]\right) \mapsto X \circ\left[\begin{array}{c}
\check{w}_{1} \\
\check{w}_{2}
\end{array}\right]:=X^{t}\left[\begin{array}{c}
\check{w}_{1} \\
\check{w}_{2}
\end{array}\right] .
$$

Consider the bases $b, \check{b}$ of $T_{p}\left(A_{0}\right), T_{p}\left(\check{A}_{0}\right)$ corresponding to

$$
\left[\begin{array}{l}
0 \\
v
\end{array}\right],\left[\begin{array}{l}
v \\
0
\end{array}\right]=\left[\begin{array}{ll}
0 & 1 \\
d & 0
\end{array}\right]\left[\begin{array}{l}
0 \\
v
\end{array}\right]
$$

and

$$
\left[\begin{array}{l}
0 \\
\check{v}
\end{array}\right],\left[\begin{array}{c}
\mathrm{d} \check{v} \\
0
\end{array}\right]=\left[\begin{array}{ll}
0 & d \\
1 & 0
\end{array}\right]\left[\begin{array}{l}
0 \\
\check{v}
\end{array}\right]=\left[\begin{array}{ll}
0 & 1 \\
d & 0
\end{array}\right] \circ\left[\begin{array}{l}
0 \\
\check{v}
\end{array}\right]
$$

Claim. One can choose $v$ and $\check{v}$ such that $b$ and $\breve{b}$ correspond to each other via $\theta_{0}$. Assuming the Claim for a moment note that, with respect to these bases, the Serre-Tate matrix of $A$ has clearly the form $\left[\begin{array}{cc}q & 0 \\ 0 & q^{d}\end{array}\right]$ and this will close the proof.

To end the proof, we must check the Claim above. First note that the involution + on $\mathcal{O}_{D}$ induces an involution + on $\operatorname{Mat}_{2}\left(\mathbf{Z}_{p}\right)$

$$
X \mapsto X^{+}=\left[\begin{array}{cc}
0 & 1 \\
-d & 0
\end{array}\right] \cdot X^{\prime} \cdot\left[\begin{array}{cc}
0 & 1 \\
-d & 0
\end{array}\right]^{-1}
$$


and the matrices below are fixed by this involution:

$$
\begin{aligned}
& {\left[\begin{array}{ll}
1 & 0 \\
0 & 0
\end{array}\right]^{+}=\left[\begin{array}{ll}
1 & 0 \\
0 & 0
\end{array}\right],} \\
& {\left[\begin{array}{ll}
0 & 1 \\
d & 0
\end{array}\right]^{+}=\left[\begin{array}{ll}
0 & 1 \\
d & 0
\end{array}\right] .}
\end{aligned}
$$

Now note that the basis $b$ is the unique basis (up to multiplication by a unit in $\mathbf{Z}_{p}$ ) having the form

$$
\left(x,\left[\begin{array}{ll}
0 & 1 \\
d & 0
\end{array}\right] x\right)
$$

with $x$ a basis of

$$
\operatorname{Ker}\left(\left[\begin{array}{ll}
1 & 0 \\
0 & 0
\end{array}\right]: T_{p}\left(A_{0}\right) \rightarrow T_{p}\left(A_{0}\right)\right) \text {. }
$$

Similarly $\breve{b}$ is the unique basis (up to multiplication by a unit in $\mathbf{Z}_{p}$ ) having the form

$$
\left(\check{x},\left[\begin{array}{ll}
0 & 1 \\
d & 0
\end{array}\right] \check{x}\right)
$$

with $\check{x}$ a basis of

$$
\operatorname{Ker}\left(\left[\begin{array}{ll}
1 & 0 \\
0 & 0
\end{array}\right]: T_{p}\left(\check{A}_{0}\right) \rightarrow T_{p}\left(\check{A}_{0}\right)\right) .
$$

Let $\theta_{0}: T_{p}\left(A_{0}\right) \rightarrow T_{p}\left(\check{A}_{0}\right)$ be the isomorphism induced by $\theta_{0}$. To conclude the proof of the Claim it is enough to check that

$$
\begin{aligned}
& {\left[\begin{array}{ll}
1 & 0 \\
0 & 0
\end{array}\right] \theta_{0} x=0,} \\
& \theta_{0}\left[\begin{array}{ll}
0 & 1 \\
d & 0
\end{array}\right] x=\left[\begin{array}{ll}
0 & 1 \\
d & 0
\end{array}\right] \theta_{0} x .
\end{aligned}
$$

By (2.28), (2.29) it is enough to check that

$$
\theta_{0} X^{+} x=X \theta_{0} x \quad\left(X \in \operatorname{Mat}_{2}\left(\mathbf{Z}_{p}\right)\right)
$$

on $T_{p}\left(A_{0}\right)$. But this is, of course, a consequence of the identity

$$
\theta_{0} \circ i\left(\alpha^{+}\right)=i(\alpha)^{\llcorner} \circ \theta_{0}: A_{0} \rightarrow \check{A}_{0}
$$

holding for all $\alpha \in \mathcal{O}_{D}$. This ends the proof of the Claim and hence of the Lemma.

It is convenient to introduce one more definition: by a $D$-frame we will understand a tuple

$$
\left(A_{0}, i_{0}, \theta_{0}, b, \check{b}\right)
$$


where $\left(A_{0}, i_{0}, \theta_{0}\right)$ is an ordinary polarized false elliptic curve over $k$ and $b, \check{b}$ satisfy the conclusion of Lemma 2.5.

A $D$-frame will be called of Jacobian type if $\left(A_{0}, \theta_{0}\right)=\left(\operatorname{Jac}\left(C_{0}\right), \theta_{C_{0}}\right)$ for some curve $C_{0}$. The following Lemma, together with Lemma 2.5, guarantees that, for all except finitely many $p$, there exists a $D$-frame of Jacobian type.

LEMMA 2.6. For all but finitely many primes $p$ one can find a polarized false elliptic curve $\left(A_{0}, i_{0}, \theta_{0}\right)$ over $k=\overline{\mathbf{F}}_{p}$ such that $A_{0}$ is ordinary and $\left(A_{0}, \theta_{0}\right)$ is isomorphic, as a polarized Abelian variety, to the Jacobian of a curve $C_{0} / k$.

We expect that the above is true for all primes $p$, or at least for all primes except those belonging to a small explicit list.

Proof. We begin by recalling the following well known fact:

FACT 1. Any principally polarized Abelian surface over $\mathbf{C}$ is isomorphic (as a polarized Abelian variety) to a Jacobian or to a product of elliptic curves.

Next we need the following:

FACT 2. Let $(A, i)$ be a false elliptic curve over $\mathbf{C}$; if $A$ is isomorphic to a product of elliptic curves $E_{1} \times E_{2}$ then both $E_{1}$ and $E_{2}$ have complex multiplication.

Indeed, assume $E_{1}$ is not $\mathrm{CM}$. If $E_{2}$ is isogenous to $E_{1}$ then $E_{2}$ is not $\mathrm{CM}$ and hence $\operatorname{End}\left(E_{1} \times E_{2}\right) \otimes \mathbf{Q} \simeq \operatorname{Mat}_{2}(\mathbf{Q})$. But there is no ring homomorphism $D \rightarrow$ $\operatorname{Mat}_{2}(\mathbf{Q})$, contradiction. So $E_{2}$ is not isogenous to $E_{1}$. But then $\operatorname{End}\left(E_{1} \times E_{2}\right) \otimes \mathbf{Q}$ is a commutative ring and, again, there cannot be any ring homomorphism from $D$ to this ring. Fact 2 is proved.

Let us now start the argument for the proof of our Lemma. Let $\mathcal{A} \rightarrow \mathcal{X}$ be the universal family of false elliptic curves (with some level structure) over some Shimura curve $\mathcal{X} / \mathbf{Z}[1 / N]$; cf. [12]. Then the geometric generic fiber of $\mathcal{A} \rightarrow \mathcal{X}$ is a Jacobian. (Indeed, if this were not the case, then, by Facts 1 and 2, this geometric generic fiber would be a product of CM elliptic curves. Since CM curves cannot 'vary in a family' we would get that all fibers of $\mathcal{A} \rightarrow \mathcal{X}$ over $\mathbf{C}$-points of $\mathcal{X}$ would be isomorphic, a contradiction.) It follows that there exists a finite covering $\mathcal{X}^{\prime}$ of a Zariski open set of $\mathcal{X}$ such that $\mathcal{A}^{\prime}:=\mathcal{A} \times \mathcal{X}^{\prime} \mathcal{X}^{\prime}$ is isomorphic (as a polarized Abelian scheme) to the Jacobian of a curve $\mathcal{C} / \mathcal{X}^{\prime}$. Since the image of $\mathcal{X}^{\prime} \rightarrow$ Spec $\mathbf{Z}$ contains an open set it follows that for all except finitely many primes $p$ we have $\mathcal{X}^{\prime} \otimes \mathbf{F}_{p} \neq \emptyset$. Hence, for any such $p$ there exists a Zariski open set $\mathcal{U}_{p} \subset \mathcal{X} \otimes \mathbf{F}_{p}$ such that for any $\overline{\mathbf{F}}_{p}$-point $x$ of $\mathcal{U}_{p}$ the corresponding principally polarized Abelian variety $\mathcal{A}_{x}$ is isomorphic to a Jacobian. Since, as well known, for all but finitely many $\overline{\mathbf{F}}_{p}$-points $x$ of $\mathcal{X} \otimes \mathbf{F}_{p}, \mathcal{A}_{x}$ is also ordinary, our Lemma is proved. 
Assume we have fixed a $D$-frame. We associate to it a homomorphism (called Serre-Tate expansion map),

$$
E_{A_{0}, D}: M_{D}^{\infty}:=\bigcup_{n} M_{D}^{n} \rightarrow S_{\mathrm{def}, D}^{\infty}:=\bigcup_{n} S_{\mathrm{def}, D}^{n}
$$

where

$$
S_{\mathrm{def}, D}^{n}:=R[[T]]\left[T^{\prime}, \ldots, T^{(n)}\right]^{\wedge} ;
$$

we define $E_{A_{0}, D}$ through the formula:

$$
E_{A_{0}, D}(f):=f\left(A_{\mathrm{def}, D}, i_{\mathrm{def}, D}, \theta_{\mathrm{def}, D}, \omega_{\mathrm{def}, D}, S_{\mathrm{def}, D}^{*}\right),
$$

where $\omega_{\text {def, } D}$ is the pull back of $\omega_{\text {def }}$ via the map

$$
\begin{aligned}
& \rho: \operatorname{Spf} S_{\text {def }, D} \rightarrow \operatorname{Spf} S_{\text {def }}, \\
& t_{11} \mapsto T, t_{22} \mapsto(T+1)^{d}-1, t_{12}, t_{21} \mapsto 0 .
\end{aligned}
$$

Note that $\omega_{\text {def, } D}$ is an invertible false 1-form, as one can check using Equations (2.3) and (2.21). We have the following Serre-Tate expansion principle on $D$ whose proof is entirely similar to the proof of Theorem 2.1 and will therefore be omitted. (The moduli spaces of Abelian schemes in the proof of the Fourier expansion principle in [2] need to be replaced, of course, by Shimura curves; a reference for the relevant facts on Shimura curves that need to be used is, for instance, [12].)

THEOREM 2.7. (1) The restriction of the Serre-Tate expansion map

$$
E_{A_{0}, D}: M_{D}^{\infty} \rightarrow S_{\mathrm{def}, D}^{\infty}
$$

to any space $M_{D}(w)$ is injective with torsion free cokernel.

(2) The induced maps

$$
M_{D}(w) \otimes_{\mathbf{Z}_{p}} R \rightarrow S_{\mathrm{def}, D}^{\infty}
$$

are injective.

The following two Propositions follow directly from the definitions:

PROPOSITION 2.8. The following diagram is commutative

$$
\begin{array}{rlc}
M_{2,1}^{\infty} & \stackrel{E_{A_{0}}}{\longrightarrow} & S_{\text {def }}^{\infty} \\
\operatorname{ind}_{D} \downarrow & & \downarrow \rho \\
M_{D}^{\infty} & \stackrel{E_{A_{0}, D}}{\longrightarrow} & S_{\mathrm{def}, D}^{\infty}
\end{array}
$$

where the left vertical arrow is as in Proposition 1.3 and the right vertical arrow is as in (2.33). 
PROPOSITION 2.9. For any $f \in I_{2}\left(\phi^{a}, \phi^{b}\right)$ we have

$$
E_{A_{0}, D}\left(\operatorname{ind}_{D} f\right) \cdot\left[\begin{array}{ll}
1 & 0 \\
0 & d
\end{array}\right]=\left[\begin{array}{ll}
\rho E_{A_{0}}\left(f_{11}\right) & \rho E_{A_{0}}\left(f_{12}\right) \\
\rho E_{A_{0}}\left(f_{21}\right) & \rho E_{A_{0}}\left(f_{22}\right)
\end{array}\right]
$$

where $\operatorname{ind}_{D}$ above is as in Proposition 1.4 and $E_{A_{0}}, E_{A_{0}, D}$ are as in Proposition 2.8.

Let $\phi^{*} \Psi$ be a collection of variables $\phi^{r} \Psi$ and consider the injective homomorphism

$$
\begin{aligned}
& \mathbf{Z}_{p}\left[\phi^{*} \Psi\right] \rightarrow S_{\mathrm{def}, D}^{n}:=R[[T]]\left[T^{\prime}, \ldots, T^{(n)}\right]^{\wedge}, \\
& \phi^{r} \Psi \mapsto \frac{1}{p} \phi^{r} \log \frac{\phi(1+T)}{(1+T)^{p}} .
\end{aligned}
$$

Then if $\Psi_{i j}$ in (2.10) are viewed as elements of $S_{\text {def }}^{\infty}$ then, clearly,

$$
\rho\left(\Psi_{11}\right)=\Psi, \quad \rho\left(\Psi_{22}\right)=\mathrm{d} \Psi, \quad \rho\left(\Psi_{12}\right)=\rho\left(\Psi_{21}\right)=0 .
$$

Recall from Remark 1.5 that we defined $f_{D}^{a}:=\operatorname{ind}_{D} f^{a} \in I_{D}\left(1+\phi^{a}\right) \subset M_{D}^{\infty}$; from Proposition 2.9 and Lemma 2.4 we get the following:

COROLLARY 2.10. Assume $\left(A_{0}, i_{0}, \theta_{0}, b, \check{b}\right)$ is a D-frame of Jacobian type. The following formula holds:

$$
E_{A_{0}, D}\left(f_{D}^{a}\right)=\epsilon\left(\phi^{a-1} \Psi+p \phi^{a-2} \Psi+\cdots+p^{a-1} \Psi\right) .
$$

In particular the map $E_{A_{0}, D}: M_{2,1}^{\infty} \rightarrow S_{\text {def }}^{\infty}$ induces a map

$$
E_{A_{0}, D}: J_{D} \rightarrow \mathbf{Z}_{p}\left[\phi^{*} \Psi\right] .
$$

Let $\phi^{*} \Lambda$ be a family of variables $\phi^{r} \Lambda, r \geqslant 0$; then the map (2.35) induces a map

$$
\begin{aligned}
& E_{A_{0}, D}^{\Lambda}: J_{D} \rightarrow \mathbf{Z}_{p}\left[\phi^{*} \Psi, \phi^{*} \Lambda\right], \\
& \sum_{w} \varphi_{w} \mapsto \sum E_{A_{0}, D}\left(\varphi_{w}\right) \epsilon^{\operatorname{deg}(w) / 2} \Lambda^{-w},
\end{aligned}
$$

where $\varphi_{w} \in J_{D}(w)$ and, for $w=\sum a_{i} \phi^{i}$, we set

$$
\Lambda^{w}:=\prod_{i}\left(\phi^{i} \Lambda\right)^{a_{i}} .
$$

In view of the Serre-Tate expansion principle for $D$ (Theorem 2.7) we get that the map (2.36) is injective.

Similarly the map

$$
E_{\infty}: J_{1} \rightarrow \mathbf{Z}_{p}\left[\phi^{*} \Psi\right]
$$


in $2.16\left(\Psi:=\Psi_{11}\right)$ gives rise to a map

$$
\begin{aligned}
E_{\infty}^{\Lambda}: J_{1} & \rightarrow \mathbf{Z}_{p}\left[\phi^{*} \Psi, \phi^{*} \Lambda\right], \\
\sum_{w} f_{w} & \mapsto \sum_{w} E_{\infty}\left(f_{w}\right) \Lambda^{-w}
\end{aligned}
$$

In view of the Fourier expansion principle for $g=1$ [7], Proposition 7.21 (or [2], Theorem 3.1), we get that the map 2.37 is injective.

Proof of Theorem 1.6. By Corollary 2.10 and by Equation (2.18) we have

$$
E_{\infty}\left(f^{a}\right)=\left(\phi^{a-1} \Psi+\cdots+p^{a-1} \Psi\right) \Lambda^{1+\phi^{a}}=E_{A_{0}, D}\left(\varphi^{a}\right) .
$$

We conclude that

$$
E_{\infty}^{\Lambda}\left(J_{1}\right)=E_{A_{0}, D}^{\Lambda}\left(J_{D}\right) .
$$

Due to the injectivity of the maps (2.36) and (2.37) we conclude that $J_{D} \simeq J_{1}$ by a $\phi$-equivariant isomorphism.

\section{Functional Equations}

\subsection{CONSTRUCTING ISOGENIES VIA SERRE-TATE PARAMETERS}

Assume we are given a $g$-frame:

$$
\left(A_{0}, \theta_{0}, b, \check{b}\right)
$$

cf. Equation (2.4). By an additional datum we will understand a tuple

$$
\left(u_{0}, A_{1}, \theta_{1}\right) \text {, }
$$

where $u_{0} \in \operatorname{End}\left(A_{0} / k\right)$ is an endomorphism and $\left(A_{1}, \theta_{1}\right)$ is a principally polarized Abelian scheme over $R$, lifting $\left(A_{0}, \theta_{0}\right)$. In particular the Serre-Tate matrix

$$
q\left(A_{1}\right):=q\left(A_{1} / R ; b, \check{b}\right)
$$

is symmetric. Let + denote the Rosati involution on $\operatorname{End}\left(A_{0} / k\right)$ (with respect to $\theta_{0}$ ) and set

$$
u_{0} b=\mu b, \quad u_{0}^{+} b=\mu^{+} b,
$$

where $b$ is viewed, as usual, as a column vector and $\mu, \mu^{+} \in \operatorname{Mat}_{g}\left(\mathbf{Z}_{p}\right)$. Note that if $\check{u}_{0} \in \operatorname{End}\left(\check{A}_{0} / k\right)$ is the dual of $u_{0}$ then

$$
\check{u}_{0} \check{b}=\check{u}_{0} \theta_{0} b=\theta_{0} u_{0}^{+} b=\theta_{0}\left(\mu^{+} b\right)=\mu^{+} \check{b} .
$$


Let $\log : 1+p R \rightarrow p R$ be the usual logarithm map. If we assume that $\mu$ is invertible $\left(\mu \in \mathrm{GL}_{g}\left(\mathbf{Z}_{p}\right)\right)$ and the matrix

$$
l:=\mu^{-1} \cdot \log q\left(A_{1}\right) \cdot\left(\mu^{+}\right)^{t}
$$

is symmetric, then the lifting $A_{2} / R$ of $A_{0} / k$ for which

$$
\log q\left(A_{2}\right):=\log q\left(A_{2} / R ; b, \check{b}\right)
$$

equals $l$ in (3.4) carries a (unique) principal polarization $\theta_{2}$ lifting $\theta_{0}$. The equality $l=\log q\left(A_{2}\right)$, together with formulas (3.2) and (3.3), immediately imply that

$$
\log q\left(A_{1} / R ; b,\left(\check{u}_{0} \check{b}\right)^{t}\right)=\log q\left(A_{2} / R ; u_{0} b, \check{b}^{t}\right) .
$$

By [19], p. 149, and the latter formula, we conclude that:

LEMMA 3.1. Assume $\mu$ is invertible and $l$ is symmetric; then $u_{0}$ lifts to a (unique) homomorphism $u: A_{1} \rightarrow A_{2}$.

\section{Let}

$$
\check{b}_{0}: A_{0}^{\text {for }} \rightarrow\left(\mathbf{G}_{m}^{\text {for }}\right)^{g}, \quad \check{b}_{1}: A_{1}^{\text {for }} \rightarrow\left(\mathbf{G}_{m}^{\text {for }}\right)^{g}, \quad \check{b}_{2}: A_{2}^{\text {for }} \rightarrow\left(\mathbf{G}_{m}^{\text {for }}\right)^{g}
$$

be the isomorphisms of formal groups that correspond to $\breve{b}$ via the functorial isomorphisms (2.2) for $A_{0}, A_{1}$ and $A_{2}$ respectively. (Here $\check{b}_{2}$ is only defined, of course, if $\mu$ is invertible and $l$ is symmetric.) Also consider the naturally induced column vectors of forms $\omega_{0}, \omega_{1}$ and $\omega_{2}$ on $A_{0}, A_{1}$ and $A_{2}$ respectively, given on the formal groups by

$$
\omega_{0}=\check{b}_{0}^{*} \frac{\mathrm{d} T}{T}, \quad \omega_{1}=\check{b}_{1}^{*} \frac{\mathrm{d} T}{T}, \quad \omega_{2}=\check{b}_{2}^{*} \frac{\mathrm{d} T}{T}
$$

where $\mathrm{d} T / T$ is the standard column of 1-forms on the torus. (Again, $\omega_{2}$ is only defined if $\mu$ is invertible and $l$ is symmetric.) We claim that the following formulae hold:

$$
u_{0}^{*} \omega_{0}=\mu^{+} \omega_{0}, \quad\left(u_{0}^{+}\right)^{*} \omega_{0}=\mu \omega_{0} .
$$

In other words,

$$
\left[u_{0}\right]=\mu^{+}, \quad\left[u_{0}^{+}\right]=\mu .
$$

Moreover, if $\mu$ is invertible and $l$ is symmetric we claim that the following formulae hold:

$$
u^{*} \omega_{2}=\mu^{+} \omega_{1}, \quad\left(u^{t}\right)^{*} \omega_{1}=\mu \omega_{2} .
$$

In other words,

$$
[u]=\mu^{+}, \quad\left[u^{t}\right]=\mu .
$$


Note that if we prove the first equality in each of (3.8), (3.9) then the second one will follow from 'symmetry'. To check the first equality in (3.8), (3.9) start by considering the following commutative diagram:

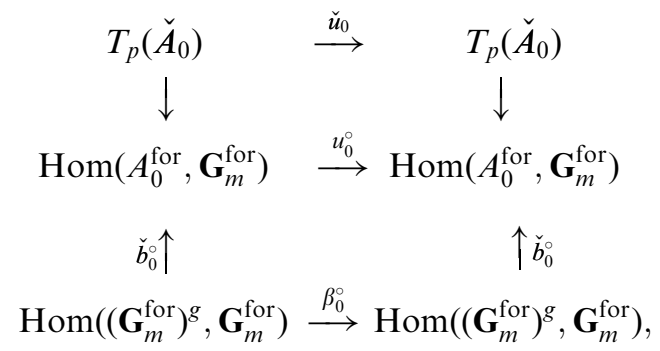

where the vertical arrow in the upper square are the isomorphisms (2.2), and the lower square is deduced by applying the functor $\operatorname{Hom}\left(-, \mathbf{G}_{m}^{\text {for }}\right)$ to the commutative square (over $k$ ):

$$
\begin{array}{ccc}
A_{0}^{\text {for }} & \stackrel{u_{0}}{\longleftarrow} & A_{0}^{\text {for }} \\
\check{b}_{0} \uparrow & & \uparrow_{\check{b}_{0}} \\
\left(\mathbf{G}_{m}^{\text {for }}\right)^{g} & \stackrel{\beta_{0}}{\longleftarrow} & \left(\mathbf{G}_{m}^{\text {for }}\right)^{g} .
\end{array}
$$

Here $\beta_{0}$ is simply the unique morphism that makes the last diagram commute. If, in addition, $\mu$ is invertible and $l$ is symmetric, the commutativity of the diagram (3.11) implies the commutativity of a diagram (over $R$ ):

$$
\begin{array}{ccc}
A_{2}^{\text {for }} & \stackrel{u}{\longleftarrow} & A_{1}^{\text {for }} \\
\check{b}_{1} \downarrow & & \downarrow^{\check{b}_{2}} \\
\left(\mathbf{G}_{m}^{\text {for }}\right)^{g} & \stackrel{\beta}{\longleftarrow} & \left(\mathbf{G}_{m}^{\text {for }}\right)^{g},
\end{array}
$$

where $\beta$ is uniquely determined by $\beta_{0}$. Now the basis $\check{b}$ in (3.10) corresponds, via the vertical isomorphisms there, to the basis of $\operatorname{Hom}\left(\left(\mathbf{G}_{m}^{\text {for }}\right)^{g}, \mathbf{G}_{m}^{\text {for }}\right)$ consisting of the $g$ canonical projections; let $P$ be the vector of these projections. Since $\check{u}_{0} \breve{b}=\mu^{+} \breve{b}$ (cf. (3.3)) it follows that $\beta_{0}^{\circ} P=\mu^{+} P$. It follows that, on the level of forms $\beta_{0}$ acts as

$$
\beta_{0}^{*} \frac{\mathrm{d} T}{T}=\mu^{+} \frac{\mathrm{d} T}{T} .
$$

We get

$$
u_{0}^{*} \omega_{0}=u_{0}^{*} \check{b}_{0}^{*} \frac{\mathrm{d} T}{T}=\check{b}_{0}^{*} \beta_{0}^{*} \frac{\mathrm{d} T}{T}=\mu^{+} \omega_{0},
$$


which proves (3.8). Assuming, in addition, that $\mu$ is invertible and $l$ is symmetric, we get that $\beta$ acts on forms as

$$
\beta^{*} \frac{\mathrm{d} T}{T}=\mu^{+} \frac{\mathrm{d} T}{T},
$$

hence we can compute

$$
u^{*} \omega_{2}=u^{*} \check{b}_{2}^{*} \frac{\mathrm{d} T}{T}=\check{b}_{1}^{*} \beta^{*} \frac{\mathrm{d} T}{T}=\check{b}_{1}^{*} \mu^{+} \frac{\mathrm{d} T}{T}=\mu^{+} \omega_{1},
$$

which proves (3.9).

As a consequence of (3.8) and (1.4) note that

$$
\operatorname{deg}\left(u_{0}\right)^{2}=\left(\operatorname{det}\left(\mu \mu^{+}\right)\right)^{2} .
$$

In particular we get that $\operatorname{deg}\left(u_{0}\right)$ is prime to $p$ if and only if $\mu$ and $\mu^{+}$are invertible. Also, if $\mu$ is invertible and $l$ is symmetric, we get

$$
\operatorname{deg}(u)^{2}=\left(\operatorname{det}\left(\mu \mu^{+}\right)\right)^{2} .
$$

\subsection{THE CASE $u_{0}=\pi-n$}

Assume again that we are given a $g$-frame (2.4) and the additional datum (3.1). Assume $A_{0}$ is defined over the finite field $\mathbf{F}_{p^{v}}$ of size $p^{v}$ and let $\pi=\pi_{v} \in \operatorname{End}\left(A_{0} / k\right)$ be the $p^{v}$-power $k$-linear Frobenius endomorphism on $A_{0}$; so if $A_{0}=A_{0, \mathbf{F}_{p^{v}}} \otimes_{\mathbf{F}_{p^{v}}} k$ then

$$
\pi_{v}=\text { (absolute } p^{v}-\text { power Frobenius) } \otimes_{\mathbf{F}_{p^{v}}} k .
$$

Recall that $\pi \pi^{+}=\pi^{+} \pi=p^{v}$ [23]. Let us assume that

$$
u_{0}=\pi-n,
$$

for some $n \in \mathbf{Z}$. Denote by $\eta, \eta^{+} \in \operatorname{Mat}_{g}\left(\mathbf{Z}_{p}\right)$ the matrices with the property that $\pi b=\eta b, \pi^{+} b=\eta^{+} b$. Then $\mu=\eta-n$ and $\mu^{+}=\eta^{+}-n$. Note that $\eta \eta^{+}=p^{v}$. We claim that $\eta$ is invertible:

$$
\eta \in \mathrm{GL}_{g}\left(\mathbf{Z}_{p}\right) \text {. }
$$

Indeed it is enough to check that $\pi$ is an isomorphism on $T_{p}\left(A_{0}\right)$, equivalently that $\check{\pi}$ is an isomorphism on $\operatorname{Hom}\left(\breve{A}_{0}^{\text {for }}, \mathbf{G}_{m}^{\text {for }}\right)$. But, since $A_{0}$ is ordinary, $\check{\pi}$ is étale, hence it induces an isomorphism on $\check{A}_{0}^{\text {for }}$ and we are done.

Note that if the $g$-frame (2.4) comes from a $D$-frame then $u_{0}=\pi-n$, being in the center of $\operatorname{End}\left(A_{0} / k\right)$, is an isogeny of false elliptic curves. If, in addition, $\mu$ is invertible and $l$ is symmetric, we have

$$
\partial(u)=\partial\left(u_{0}\right)=\mu \mu^{+} .
$$

\subsection{MULTIPLICATION BY $\mathcal{O}_{L}$}

Let $L$ be a simple central Q-algebra of dimension $g^{2}$ and let $\mathcal{O}_{L}$ be an order in $L$, stable under a given involution + on $L$. Say that $\left(A_{0}, \theta_{0}\right)$ has multiplication by $\mathcal{O}_{L}$ 
if there is a + equivariant injection $i_{0}: \mathcal{O}_{L} \rightarrow \operatorname{End}\left(A_{0} / k\right)$ (where + acts on endomorphisms as the Rosati involution). We are especially interested in the following two situations:

(1) $\left(A_{0}, i_{0}, \theta_{0}\right)$ is a polarized false elliptic curve. In this case $L=D$ is our indefinite quaternion algebra with its involution + .

(2) $A_{0}$ is the $g$-fold power of an elliptic curve $E_{0}$ and $\theta_{0}$ is the $g$-fold power of the canonical polarization on $E_{0}$. In this case $\mathcal{O}_{L}=\operatorname{Mat}_{g}(\mathbf{Z})$ with the involution + given by transposition.

LEMMA 3.2. Fix a g-frame $\left(A_{0}, \theta_{0}, b, \check{b}\right)$ and assume $\left(A_{0}, \theta_{0}\right)$ has multiplication by $\mathcal{O}_{L}$. Then $\eta, \eta^{+}$are scalar; so $\eta \in \mathbf{Z}_{p}^{\times}$and $\eta^{+} \in p^{v} \mathbf{Z}_{p}$.

Proof. The map $i_{0}$ composed with the natural representation

$$
\operatorname{End}\left(A_{0} / k\right) \rightarrow \operatorname{End}\left(T_{p}\left(A_{0}\right)\right)
$$

and the isomorphism

$$
\operatorname{End}\left(T_{p}\left(A_{0}\right)\right) \simeq \operatorname{Mat}_{g}\left(\mathbf{Z}_{p}\right)^{o p}
$$

induced by the basis $b$ induces an algebra map

$$
L \otimes_{\mathbf{Q}} \mathbf{Q}_{p} \rightarrow \operatorname{Mat}_{g}\left(\mathbf{Q}_{p}\right)^{o p} .
$$

Since $L \otimes_{\mathbf{Q}} \mathbf{Q}_{p}$ is simple and central the map (3.17) is an isomorphism. Since $\pi, \pi^{+}$ are in the center of $\operatorname{End}\left(A_{0} / k\right), \eta, \eta^{+}$commute with all elements in the image of $\mathcal{O}_{L}$ in $\operatorname{Mat}_{g}\left(\mathbf{Q}_{p}\right)$, hence they must commute with all elements of $\operatorname{Mat}_{g}\left(\mathbf{Q}_{p}\right)$, hence they are scalar, which ends our proof.

\subsection{FUNCTIONAL EQUATION SATISFIED BY SERRE-TATE EXPANSIONS}

Assume we are in the hypotheses of Lemma 3.2 and assume $u_{0}=\pi-n$ as in 3.14 with

$$
n \not \equiv 0, \eta \bmod p .
$$

Then $\mu, \mu^{+} \in \mathbf{Z}_{p}^{\times}$, hence, in particular, $\operatorname{deg}\left(u_{0}\right)$ is prime to $p$ and $l$ in (3.4) is obviously symmetric. So $A_{2}$ and $u: A_{1} \rightarrow A_{2}$ are defined, cf. Lemma 3.1. Define

$$
\gamma=\gamma_{n}:=\frac{\mu^{+}}{\mu}=\frac{\eta^{+}-n}{\eta-n} \in \mathbf{Z}_{p}^{\times} .
$$

The equality between (3.4) and (3.5) implies that

$$
q_{i j}\left(A_{2}\right)=q_{i j}\left(A_{1}\right)^{\gamma} .
$$

Note that if our $g$-frame comes from a $D$-frame and if $A_{1}$ is the false elliptic curve with matrix

$$
q\left(A_{1}\right)=\left[\begin{array}{cc}
q & 0 \\
0 & q^{d}
\end{array}\right]
$$


cf. Lemma 2.5, for some $q \in 1+p R$, then

$$
q\left(A_{2}\right)=\left[\begin{array}{cc}
q^{\gamma} & 0 \\
0 & \left(q^{d}\right)^{\gamma}
\end{array}\right]=\left[\begin{array}{cc}
q^{\gamma} & 0 \\
0 & \left(q^{\gamma}\right)^{d}
\end{array}\right],
$$

so $A_{2}$ will be naturally a false elliptic curve (call $i_{2}$ the corresponding structure), by the same Lemma 2.5 .

LEMMA 3.3. Let $\left(A_{0}, i_{0}, \theta_{0}, b, \breve{b}\right)$ be a $D$-frame, let $u_{0}=\pi-n$ as in (3.14) with $n \not \equiv 0, \eta \bmod p$, cf. (3.18), and let $\gamma_{n}$ be defined by Equation (3.19). Let $f \in I_{D}(w)$ be a $\delta$-modular form on $D$ of weight $w$. Then

$$
f\left(A_{2}, i_{2}, \theta_{2}, \omega_{2}, R^{*}\right)=\gamma^{-\frac{\operatorname{deg}(\omega)}{2}} f\left(A_{1}, i_{1}, \theta_{1}, \omega_{1}, R^{*}\right) .
$$

Proof. We have the following computation, using Equations (1.18), (3.16), and (3.9):

$$
\begin{aligned}
f\left(A_{2}, i_{2}, \theta_{2}, \omega_{2}, R^{*}\right) & =\left(\mu \mu^{+}\right)^{\frac{\operatorname{deg}(w)}{2}} f\left(A_{1}, i_{1}, \theta_{1}, u^{*} \omega_{2}, R^{*}\right) \\
& =\left(\mu \mu^{+}\right)^{\frac{\operatorname{deg}(w)}{2}} \chi_{w}\left(\mu^{+}\right)^{-1} f\left(A_{1}, i_{1}, \theta_{1}, \omega_{1}, R^{*}\right) \\
& =\gamma^{-\frac{\operatorname{deg}(w)}{2}} f\left(A_{1}, i_{1}, \theta_{1}, \omega_{1}, R^{*}\right) .
\end{aligned}
$$

LEMMA 3.4. The ring homomorphism

$$
\begin{aligned}
& \sigma: R\left[\left[T, T^{\prime}, \ldots, T^{(n)}\right]\right] \rightarrow R\left[\left[T, T^{\prime}, \ldots, T^{(n)}\right]\right], \quad F \mapsto \tilde{F}, \\
& \tilde{F}\left(T, T^{\prime}, \ldots, T\right):=F\left(p T, \delta(p T), \ldots, \delta^{n}(p T)\right)
\end{aligned}
$$

is injective.

Here $\delta: R\left[\left[T, T^{\prime}, \ldots, T^{(i)}\right]\right] \rightarrow R\left[\left[T, T^{\prime}, \ldots, T^{(i+1)}\right]\right]$ are the unique $p$-derivations sending $T \mapsto T^{\prime}, T \mapsto T^{\prime \prime}$, etc.

Proof. Note that $\sigma$ in our Lemma extends to a ring homomorphism

$$
\Sigma: K\left[\left[T, T^{\prime}, \ldots, T^{(n)}\right]\right] \rightarrow K\left[\left[T, T^{\prime}, \ldots, T^{(n)}\right]\right]
$$

defined by the same formula as $\sigma$. Now one can prove by induction that

$$
\delta^{n}(p T)=p T^{(n)}+P_{n}\left(T, \ldots, T^{(n-1)}\right), \quad P_{n} \in \mathbf{Z}\left[T, \ldots, T^{(n-1)}\right] .
$$

So $\Sigma$ is an isomorphism. Hence, $\sigma$ is injective.

Note that the map $\sigma$ in Equation (3.22) induces a map

$$
R[[T]]\left[T^{\prime}, \ldots, T^{(n)}\right]^{\wedge} \rightarrow R\left[T, T^{\prime}, \ldots, T^{(n)}\right]^{\wedge} .
$$


For $\gamma \in \mathbf{Z}_{p}$, define the series

$$
\gamma(T)=\frac{(1+p T)^{\gamma}-1}{p}=\gamma T+\gamma(\gamma-1) \frac{p}{2 !} T^{2}+\cdots \in \mathbf{Z}_{p}[T]^{\wedge} .
$$

LEMMA 3.5. Let $\left(A_{0}, i_{0}, \theta_{0}, b, \check{b}\right)$ be a $D$-frame, let $f \in I_{D}(w)$ be any $\delta$-modular form on $D$ of weight $w$, with $\operatorname{ord}(w)=n$, and let

$$
F\left(T, T^{\prime}, \ldots, T^{(n)}\right)=E_{A_{0}, D}(f) \in R[[T]]\left[T^{\prime}, \ldots, T^{(n)}\right]^{\wedge}
$$

be its Serre-Tate expansion, cf. (2.31). Then, for any $\gamma \in \mathbf{Z}_{p}$ with $\gamma \neq \equiv 0,1 \bmod p$, the series $\tilde{F}$ satisfies the functional equation:

$$
\tilde{F}\left(\gamma(T), \delta(\gamma(T)), \ldots, \delta^{n}(\gamma(T))\right)=\gamma^{\frac{-\operatorname{deg}((w)}{2}} \cdot \tilde{F}\left(T, T^{\prime}, \ldots, T^{(n)}\right)
$$

in $R\left[T, T^{\prime}, \ldots, T^{(n)}\right]^{\wedge} \subset R\left[\left[T, T^{\prime}, \ldots, T^{(n)}\right]\right]$.

Proof. Choose an additional datum $\left(u_{0}, A_{1}, \theta_{1}\right)$, cf. (3.1), where:

(1) $u_{0}=\pi-n$, cf. (3.14), with $n \not \equiv 0, \eta \bmod p$, cf. (3.18) and

(2) $A_{1} / R$ is an arbitrary lifting of $A_{0} / k$ to a false elliptic curve.

Let $\gamma_{n}$ be defined by the formula (3.19) and let $\left[\begin{array}{cc}q & 0 \\ 0 & q^{d}\end{array}\right]$ be the Serre-Tate matrix of $A_{1}$. Note that if $q=1+p x$ with $x \in R$ then $q^{\gamma}=1+p \cdot \gamma(x)$. So, due to Equation (3.21), our Equation (3.24) holds for $\gamma=\gamma_{n}$ if we replace $T$ by any $x \in R$. By [6], Lemma 2.12, we conclude that the Equation (3.24) itself holds for any $\gamma=\gamma_{n}$ as long as $n \neq \equiv, \eta \bmod$ $p$. To conclude that the Equation (3.24) holds for any $\gamma \neq \equiv 0,1 \bmod p$, fix such a $\gamma$ and take a sequence of integers $n_{i}$ converging $p$-adically to the $p$-adic number

$$
\tilde{n}:=\frac{\eta^{+}-\gamma \eta}{1-\gamma}
$$

since $\tilde{n} \not \equiv 0, \eta \bmod p$ one has that $n_{i} \not \equiv 0, \eta \bmod p$ for $i \gg 0$. It follows that $\gamma_{n} \rightarrow \gamma$, $p$-adically. Passing to the limit in our Equation (3.24) for $\gamma_{n}$ we get that Equation (3.24) holds for $\gamma$.

For $\gamma \in \mathbf{Z}_{p}$, define the series

$$
\Gamma(T)=\Gamma_{\gamma}(T)=(1+T)^{\gamma}-1=\gamma T+\frac{\gamma(\gamma-1)}{2 !} T^{2}+\cdots \in \mathbf{Z}_{p}[[T]] ;
$$

in other words,

$$
\gamma(T)=\frac{\Gamma(p T)}{p} .
$$

LEMMA 3.6. Let $\left(A_{0}, i_{0}, \theta_{0}, b, \breve{b}\right)$ be a $D$-frame, let $f \in I_{D}(w)$ be any $\delta$-modular form on $D$ of weight $w$, with $\operatorname{ord}(w)=n$, and let

$$
F\left(T, T^{\prime}, \ldots, T^{(n)}\right)=E_{A_{0}, D}(f) \in R[[T]]\left[T^{\prime}, \ldots, T^{(n)}\right]^{\wedge}
$$


be its Serre-Tate expansion, $c f$. (2.31). Then, for any $\gamma \in \mathbf{Z}_{p}$ with $\gamma \neq \equiv 0,1 \bmod p$, the series $F$ satisfies the functional equation:

$$
F\left(\Gamma(T), \delta(\Gamma(T)), \ldots, \delta^{n}(\Gamma(T))\right)=\gamma^{-\frac{\operatorname{deg}(w)}{2}} \cdot F\left(T, T^{\prime}, \ldots, T^{(n)}\right)
$$

in the ring $R\left[\left[T, T^{\prime}, \ldots, T^{(n)}\right]\right]$.

Proof. Let $G$ be the difference between the left-hand side and the right-hand side of Equation (3.26). Then, with the notations of Lemma 3.4 it is trivial to see that $\sigma(G)$ coincides with the difference between the left-hand side and the right-hand side of Equation 3.24. By Lemma 3.6, $\sigma(G)=0$. By Lemma 3.4, $G=0$.

By the way similar conclusions can be reached if $\left(A_{0}, \theta_{0}\right)$ is an elliptic curve.

LEMMA 3.7. Assume $\left(A_{0}, \theta_{0}, b, \check{b}\right)$ is a 1-frame where $\left(A_{0}, \theta_{0}\right)$ is an elliptic curve. Then, for any $\gamma \in \mathbf{Z}_{p}, \gamma \neq \equiv 0,1 \bmod p$, and any Siegel $\delta$-modular form $f \in I_{1}(w)$ of weight $w$, with $\operatorname{ord}(w)=n$, the Serre-Tate expansion

$$
F\left(T, T^{\prime}, \ldots, T^{(n)}\right)=E_{A_{0}}(f) \in R[[T]]\left[T^{\prime}, \ldots, T^{(n)}\right]^{\wedge}
$$

satisfies the functional equation

$$
F\left(\Gamma(T), \delta(\Gamma(T)), \ldots, \delta^{n}(\Gamma(T))\right)=\gamma^{\frac{-\operatorname{deg}(w)}{2}} \cdot F\left(T, T^{\prime}, \ldots, T^{(n)}\right)
$$

in the ring $R\left[\left[T, T^{\prime}, \ldots, T^{(n)}\right]\right]$.

\subsection{SOLVING THE FUNCTIONAL EQUATION}

Next we solve the functional equation appearing in Proposition 3.6. A similar solution can be given to the functional equation in Proposition 3.7.

LEMMA 3.8. Let $\gamma \in \mathbf{Z}_{p}$ and $H \in R\left[\left[T, T^{\prime}, \ldots, T^{(n)}\right]\right]^{\wedge}$. If $H \in(T)^{2}$, then

$$
\delta^{n}(\gamma T+H)-\gamma T^{(n)} \in\left(T, T^{\prime}, \ldots, T^{(n)}\right)^{2} .
$$

Proof. Induction on $n$.

Recall that we considered the variables $\phi^{i} \Psi$ identified with the series

$$
\phi^{i} \log \frac{1+\phi(T)}{(1+T)^{p}} \in R[[T]]\left[T^{\prime}\right]^{\wedge} .
$$

LEMMA 3.9. Let $m$ be an integer, let $\gamma \in \mathbf{Z}_{p}$ be any $p$-adic number which is not a root of unity and let $V=V(n, m, \gamma)$ be the $K$-vector space $(K:=R[1 / p])$ of all series $G \in K\left[\left[T, \ldots, T^{(n)}\right]\right]$ satisfying the functional equation

$$
G\left(\Gamma(T), \delta(\Gamma(T)), \ldots, \delta^{n}(\Gamma(T))\right)=\gamma^{m} \cdot G\left(T, T^{\prime}, \ldots, T^{(n)}\right) .
$$


Then $V$ consists of all series of the form

$$
\Phi\left(\log (1+T), \Psi, \phi \Psi, \ldots, \phi^{n-1} \Psi\right),
$$

where $\Phi \in K\left[x_{0}, \ldots, x_{n}\right]$ is a homogeneous polynomial of degree $m$.

Proof. The space of all series of the form (3.29) is clearly contained in $V$ and has dimension $(m+n) ! / m ! n !$. So it is enough to show that $V$ has dimension at most $(m+n) ! / m ! n !$. It is enough to show that the map that attaches to any $G \in V$ its homogeneous component of degree $m$ is injective. Let $G \in V, G=G_{v}+G_{v+1}+\cdots$, $G_{i}$ homogeneous of degree $i, G_{v} \neq 0$. Assume $G_{m}=0$ (in particular $v \neq m$ ) and seek a contradiction. By Lemma 3.8 we get

$$
G\left(\gamma X+H_{0}, \gamma X^{\prime}+H_{1}, \ldots, \gamma X^{(n)}+H_{n}\right)=\gamma^{m} \cdot G\left(X, X^{\prime}, \ldots, X^{(n)}\right),
$$

for some $H_{0}, \ldots, H_{n} \in\left(X, \ldots, X^{(n)}\right)^{2}$. Picking out the component of degree $v$ in the above equality we get

$$
\gamma^{v} \cdot G_{v}=\gamma^{m} \cdot G_{v} .
$$

We conclude that $\gamma^{v-m}=1$ which contradicts the assumption that $\gamma$ is not a root of unity.

LEMMA 3.10. Let $\Phi \in K\left[x_{0}, \ldots, x_{n}\right]$ be a homogeneous polynomial of degree $m \geqslant 1$ such that

$$
\Phi\left(\log (1+T), \Psi, \phi \Psi, \ldots, \phi^{n-1} \Psi\right) \in R[[T]]\left[T^{\prime}, \ldots, T^{(n)}\right]^{\wedge},
$$

then $\partial \Phi / \partial x_{0}=0$

Proof. First note that the Lemma is true for $m=1$ due to the fact that for any $v \in \mathbf{Z}_{+}, p^{v} \cdot \log (1+T) \notin R[[T]]$.

Now we proceed by induction on $m+n$. If $m+n=1$ we must have $m=1$ and we are done. Assume now $m+n \geqslant 2$ and let $\Phi$ be as in the statement of the Lemma. If $m=1$ we are done so we may assume $m \geqslant 2$. Write

$$
\Phi=\Phi_{0}+\Phi_{1} x_{n}+\cdots+\Phi_{s} x_{n}^{s}
$$

with $\Phi_{i} \in K\left[x_{0}, \ldots, x_{n-1}\right]$. Consider the derivation

$$
D_{n}=\left(1+\phi^{n} T\right) \cdot \frac{\partial}{\partial T^{(n)}}: R[[T]]\left[T^{\prime}, \ldots, T^{(n)}\right]^{\wedge} \rightarrow R[[T]]\left[T^{\prime}, \ldots, T^{(n)}\right]^{\wedge} .
$$

Note that $D_{n}\left(\phi^{n-1} \Psi\right)=p^{n-1}$. So we get

$$
\frac{\partial \Phi}{\partial x_{n}}\left(\log (1+T), \Psi, \phi \Psi, \ldots, \phi^{n-1} \Psi\right) \cdot p^{n-1} \in R[[T]]\left[T^{\prime}, \ldots, T^{(n)}\right]^{\wedge} .
$$

Since $\partial \Phi / \partial x_{n}$ has degree $m-1 \geqslant 1$, we get by induction that

$$
\frac{\partial^{2} \Phi}{\partial x_{0} \partial x_{n}}=0
$$


Since $m \geqslant 2$, we must have $n \neq 0$. We conclude that

$$
\frac{\partial \Phi_{1}}{\partial x_{0}}=\cdots=\frac{\partial \Phi_{s}}{\partial x_{0}}=0
$$

hence

$$
\left(\Phi_{1} x_{n}+\cdots+\Phi_{s} x_{n}^{s}\right)\left(\log (1+T), \Psi, \phi \Psi, \ldots, \phi^{n-1} \Psi\right) \in R[[T]]\left[T^{\prime}, \ldots, T^{(n)}\right]^{\wedge},
$$

hence

$$
\Phi_{0}\left(\log (1+T), \Psi, \phi \Psi, \ldots, \phi^{n-2} \Psi\right) \in R[[T]]\left[T^{\prime}, \ldots, T^{(n)}\right]^{\wedge} .
$$

By induction, we get

$$
\frac{\partial \Phi_{0}}{\partial x_{0}}=0
$$

and we are done.

THEOREM 3.11. For any series $G \in R[[T]]\left[T^{\prime}, \ldots, T^{(n)}\right]^{\wedge}$ and any integer $m \geqslant 0$ the following are equivalent:

(1) $G$ satisfies the functional Equation (3.28) for some $\gamma \in \mathbf{Z}_{p}, \gamma$ not a root of unity.

(2) $G$ satisfies the functional Equation (3.28) for all $\gamma \in \mathbf{Z}_{p}$.

(3) There exists a homogeneous polynomial $\Phi \in K\left[x_{1}, \ldots, x_{n}\right]$ of degree $m$ such that

$$
\Phi\left(\Psi, \phi \Psi, \ldots, \phi^{n-1} \Psi\right),
$$

Proof. Clearly Assertion (3) implies Assertion (2) and Assertion (2) implies assertion (1). Now if Assertion (1) holds then by Lemma 3.9, there exists a homogeneous polynomial $\Phi \in K\left[x_{0}, x_{1}, \ldots, x_{n}\right]$ of degree $m$ such that

$$
G=\Phi\left(\log (1+T), \Psi, \phi \Psi, \ldots, \phi^{n-1} \Psi\right) .
$$

By Lemma 3.10, $\Phi$ does not depend on $x_{0}$ and we are done.

THEOREM 3.12. Let $w$ be a weight with $\operatorname{deg}(w)=-2 m,(m \in \mathbf{Z}), \operatorname{ord}(w)=n$. Assume $f$ is either a form in $I_{D}(w)$ or in $I_{1}(w)$, and let $\left(A_{0}, \ldots\right)$ be either a $D$-frame or a 1-frame respectively. Then the Serre-Tate expansion $E(f)$ of $f$ correspoding to this frame has the form

$$
E(f)=\Phi\left(\Psi, \phi \Psi, \ldots, \phi^{n-1} \Psi\right),
$$

where $\Phi$ is a homogeneous polynomial of degree $m$, in $n$ variables, with coefficients in $K=R[1 / p]$.

Proof. By Lemmas 3.6 and 3.7, respectively, the series $E(f)$ belongs to the space $V(n, m, 2)$; cf. the notations in Lemma 3.9. Then we may conclude by Theorem 3.11 . 
Proof (of Assertions 1-4 in Theorem 1.2) Let $w$ be a weight with $\operatorname{ord}(w)=n$, let $f \in I_{D}(w)$, and let $\left(A_{0}, i_{0}, \theta_{0}, b, \breve{b}\right)$ be a $D$-frame. By Theorem 2.7 we have an injective map

$$
I_{D}(w) \otimes_{\mathbf{Z}_{p}} R \rightarrow R[[T]]\left[T^{\prime}, \ldots, T^{(n)}\right]^{\wedge} ;
$$

by Theorem 3.12 the image of this map is contained in the $R$-module $\mathcal{V}$ of all series in $R[[T]]\left[T^{\prime}, \ldots, T^{(n)}\right]^{\wedge}$ of the form $\Phi\left(\Psi, \phi \Psi, \ldots, \phi^{n-1} \Psi\right)$ where $\Phi \in K\left[x_{1}, \ldots, x_{n}\right]$ is homogeneous of degree $m=-\operatorname{deg}(w) / 2$. It is easy to see that there exists an integer $v \geqslant 0$ such that $p^{-v} \mathcal{V}^{\prime} \subset \mathcal{V} \subset \mathcal{V}^{\prime}$, where $\mathcal{V}^{\prime}$ is the $R$-module generated by all monomials

$$
\Psi^{i_{0}}(\phi \Psi)^{i_{1}} \ldots\left(\phi^{n-1} \Psi\right)^{i_{n-1}}, \quad i_{0}+\cdots+i_{n-1}=m .
$$

So $\mathcal{V}$ is a finitely generated $R$-module of rank $s:=(m+n-1) ! / m !(n-1)$ !. Hence $I_{D}(w) \otimes_{\mathbf{Z}_{p}} R$ is a finitely generated $R$-module of rank at most $s$. Since $R$ is faithfully flat over $\mathbf{Z}_{p}$ it follows that $I_{D}(w)$ itself is a finitely generated $\mathbf{Z}_{p}$-module of rank at most $s$; so Assertion 1 in Theorem 1.2 is proved. Note that $\mathcal{V}=0$ if $\operatorname{deg}(w)>0$ and $\mathcal{V}$ has rank one if $w \in\{0,-1-\phi\}$. This implies that $I_{D}(w)=0$ for $\operatorname{deg}(w)>0$, $I_{D}(0)=\mathbf{Z}_{p}$, and $I_{D}(-1-\phi)$ has rank one; so Assertions 2,3,4 in Theorem 1.2 are proved.

As already noted, the proof of Assertion (5) in Theorem 1.2 requires additional tools; these tools and the proof of Assertion (5) will be presented in a sequel to the present paper [9].

\section{Acknowledgement}

The author is indebted to the referee of an earlier paper for comments that were partially incorporated in the present paper. While writing this paper the author was partially supported by NSF grant DMS 0096946.

\section{References}

1. Barcau, M.: Isogeny covariant differential modular forms and the space of elliptic curves up to isogeny, Compositio Math. to appear.

2. Barcau, M. and Buium, A.: Siegel differential modular forms, Internat. Math. Res. Notices. 28 (2002), 1459-1503.

3. Boutot, J. F. and Carayol, H.: Uniformisation p-adique des courbes de Shimura; les theoremes de Cerednik at de Drinfeld, Asterisque 196-197 (1991), 45-158.

4. Buium, A.: Differential Algebra and Diophantine Geometry, Hermann, Paris, 1992.

5. Buium, A.: Differential characters of Abelian varieties over p-adic fields, Invent. Math. 122 (1995), 309-340.

6. Buium, A.: Geometry of p-jets, Duke J. Math. 82(2) (1996), 349-367.

7. Buium, A.: Differential modular forms, J. Reine Angew. Math. 520 (2000), 95-167.

8. Buium, A.: Geometry of Fermat Adeles, Preprint. 
9. Buium, A.: Differential modular forms on Shimura curves, II: Serre operators, Compositio Math., to appear.

10. Buium, A.: Quotients of algebraic varieties by Zariski dense equivalence relations, In: The Parshin Festschrift, Contemp. Math. 300, Amer. Math. Soc., Providence 2002, pp. 59-97.

11. Buium, A. and Zimmerman, K.: Differential orbit spaces of discrete dynamical systems, I, preprint.

12. Buzzard, K.: Integral models of certain Shimura curves, Duke Math J. 87(3) (1997), 591-612.

13. Connes, A.: Noncommutative Geometry, Academic Press, 1994.

14. Deligne, P. and Illusie, L.: Cristaux ordinaires et coordonnees canoniques, In: Surfaces Algebriques, Lecture Notes in Math. 686, Springer, New York, 1981.

15. Diamond, F. and Taylor, R.: Non-optimal levels for mod $l$ modular representations of $\operatorname{Gal}(\overline{\mathbf{Q}} / \mathbf{Q})$, Invent. Math. 115 (1994), 435-462.

16. Dwork, B. and Ogus, A.: Canonical liftings of Jacobians, Compositio Math. 58 (1986), 111-131.

17. Faltings, G. and Chai, C-L.: Degeneration of Abelian Varieties, Springer, Heidelberg, 1990.

18. Katz, N.: Travaux de Dwork, Expose 409, Sem. Bourbaki 1971/72, Lecture Notes in Math. 317, Springer, New York, 1973, pp. 167-200.

19. Katz, N.: Serre-Tate local moduli, In: Lecture Notes in Math. 868, Springer, New York, 1981, pp. 138-202.

20. Katz, N.: $P$-adic properties of modular schemes and modular forms, In: Lecture Notes in Math. 350, Springer, New York, 1973, pp. 69-190.

21. Kolchin, E.: Differential Algebra and Algebraic Groups, Academic Press, New York, 1973.

22. Katz, N. and Mazur, B.: Arithmetic Moduli of Elliptic Curves, Ann. of Math. Stud., Princeton Univ. Press, 1985.

23. Milne, J. S.: Abelian varieties, In: G. Cornell and J. H. Silverman (eds), Arithmetic Geometry, Springer, New York, 1986.

24. Moonen, B.: Linear properties of Shimura varieties, II, Compositio Math. 114 (1998), 3-35.

25. Mori, A.: Power series expansions of modular forms at CM points, Rend. Sem. Mat. Univ. Pol. Torino 534 (1995), 361-374.

26. Noot, R.: Models of Shimura varieties in mixed characteristic, J. Algebraic Geom. 5 (1996), 187-207.

27. Oort, F. and Steenbrink, J.: The local Torelli problem for algebraic curves, Geom. Algebrique (Angers 1979), Sijthof and Nordhoff, 1980, pp. 157-205.

28. Ritt, J. F.: Differential Algebra, AMS Collogu. Publ. 33, Amer. Math. Soc., Providence, 1950.

29. Shimura, G.: Moduli of abelian varieties and number theory, In: Algebraic Groups and Discontinuous Groups, Proc. Sympos. Pure Math., Amer. Math. Soc., Providence, 1966, pp. 312-333.

30. Weil, A.: Basic Number Theory, Springer, New York. 